\title{
Batf-dependent Th17 cells critically regulate IL-23 driven colitis-associated colon cancer
}

\author{
Elise Punkenburg, ${ }^{1,2}$ Tina Vogler, ${ }^{1,2}$ Maike Büttner, ${ }^{3}$ Kerstin Amann, $^{3}$ Max Waldner, ${ }^{1}$ \\ Raja Atreya, ${ }^{1}$ Benjamin Abendroth, ${ }^{1,2}$ Jonas Mudter, ${ }^{6}$ Susanne Merkel, ${ }^{4}$ \\ Eike Gallmeier, ${ }^{7}$ Stefan Rose-John, ${ }^{5}$ Markus F Neurath, ${ }^{1}$ Kai Hildner ${ }^{1,2}$
}

- Additional material is published online only. To view please visit the journal online (http://dx.doi.org/10.1136/ gutjnl-2014-308227).

For numbered affiliations see end of article.

\section{Correspondence to}

Professor Kai Hildner, Department of Medicine 1, University Hospital Erlangen, Ulmenweg 18, Erlangen D-91054, Germany; Kai.Hildner@uk-erlangen.de

Received 8 August 2014 Revised 1 March 2015 Accepted 9 March 2015 Published Online First 2 April 2015

\section{ABSTRACT}

Objectives IBDs have an increased risk for development of colorectal cancer (CRC). Here, we aimed at the characterisation of the functional role of Th17associated transcription factors in sporadic and colitisassociated colon cancer in vivo.

Design We used mice deficient or transgenic for the activating protein 1 family member basic leucine zipper transcription factor ATF-like (Batf) to evaluate the role of Th17 cells during sporadic and inflammation-induced colon carcinogenesis. We also studied the expression of Batf and ROR $\gamma$ t in patients with IBD and CRC.

Results Batf but not retinoic acid-related orphan receptor $\gamma t(\mathrm{ROR} \gamma \mathrm{t})$ expression was significantly increased together with interleukin (IL) 23 expression in UC but not in Crohn's disease (CD) tissue samples. In CRC also Batf but not ROR $\gamma$ t expression was increased and its expression correlated with the IL-23 and IL-23 receptor (IL-23R) expression. Finally, Batf but not RORyt was coexpressed with IL-17a, IL-23R and IL-6 within CRCinfiltrating $\mathrm{CD}^{+} \mathrm{T}$ cells. Functional studies in mice revealed that Batf-dependent T cells are crucial regulators of sporadic and inflammation-induced CRC. Colitis-associated Batf ${ }^{-1}$ tumours lacked IL-17a ${ }^{+} \mathrm{IL}-$ $23 \mathrm{R}^{+} \mathrm{IL}-6^{+} \mathrm{CD} 4^{+} \mathrm{T}$ cells, hence displaying characteristics reminiscent of human CRC-infiltrating $\mathrm{CD}^{+} \mathrm{T}$ cells. Strikingly, Batf ${ }^{-1-}$ tumours contained low IL-23 but high IL-17a expression levels. Tumour formation and intratumoral IL-23 expression could be restored by administration of Hyper-IL-6 consisting of IL-6 and soluble IL-6 receptor.

Conclusions Batf-dependent IL-23R ${ }^{+} \mathrm{IL}-6^{+} \mathrm{CD} 4^{+}$Th17 cells critically control IL-23 driven colitis-associated tumour formation and the progression of sporadic colon tumours. Batf-dependent IL-23R ${ }^{+} \mathrm{T}$ cells represent a potential future therapeutic target limiting CRC progression.

\section{INTRODUCTION}

Colorectal cancer (CRC) represents one of the most common malignancies in the western world. Epidemiological data non-ambiguously identified chronic intestinal inflammation as a predisposing condition increasing the risk of developing clinically apparent carcinoma. ${ }^{1}{ }^{2}$ Accordingly, patients with IBD, such as UC, possess a significantly increased lifetime risk of developing so-called colitis-associated cancer $(\mathrm{CAC}) .{ }^{1}$ However, the

\section{Significance of this study}

What is already known on this subject?

- Chronic mucosal inflammation predisposes patients with IBD to develop colitis-associated cancer (CAC).

- An interleukin (IL) $23^{+} / \mathrm{IL}-17 \mathrm{a}^{+}$enriched tumour microenvironment in colorectal cancer (CRC) tissue is linked to a poor prognosis. While IL-17a ${ }^{+} \mathrm{CD} 4^{+} \mathrm{T}$ cells (Th17 cells) are frequently found in CRC tissue, their selective role and contribution during tumour promotion however has not been studied in vivo.

- The transcription factors retinoic acid receptor-related orphan nuclear receptor $\gamma t$ (ROR $\gamma \mathrm{t}$ ) and the activating protein 1 family member basic leucine zipper transcription factor ATF-like (Batf) are both crucial regulators of Th17 cell differentiation rendering mice deficient for these genes uniformly refractory to the development of Th17 dependent tissue inflammation.

What are the new findings?

- Identification of Batf but not ROR $\gamma \mathrm{t}$ as a selectively upregulated Th17 marker in UC and CRC tissues; Batf expression correlated with intratumoral IL-23p19 and IL-23R expression and was increased in CRC-infiltrating IL-17a ${ }^{+} \mathrm{IL}-23 \mathrm{R}^{+} \mathrm{IL}-6^{+} \mathrm{CD} 4^{+} \mathrm{T}$ cells.

- Batf-dependent Th17 cells are shown to be critical regulators of sporadic and inflammation-induced colon cancer: Batf ${ }^{-1-}$ mice showed reduced CAC formation in the presence of abundant IL-17a expression by non-Th17 cells despite reduced intratumoral IL-23 expression in vivo while T cells overexpressing Batf promoted CAC progression.

- Batf deficiency forecloses the development of IL-23R expressing CD4 ${ }^{+}$Th17 cells in vitro and in vivo as Batf ${ }^{-1-}$ tumours lacked IL-17a ${ }^{+}$IL-23R ${ }^{+}$IL- $6^{+} \mathrm{CD} 4^{+}$T cells; reconstitution of Batf ${ }^{-l-}$ mice with Hyper-IL6, a designer cytokine consistent of IL- 6 and soluble IL-6R, restored CAC progression and IL-23 expression suggesting a central role of Batf-dependent IL- $6^{+}$Th17 cells during IL-23 driven colon tumour progression. 


\section{Significance of this study}

\section{How might it impact on clinical practice in in the foreseeable future?}

- Batf-dependent IL-17a ${ }^{+} \mathrm{IL}-23 \mathrm{R}^{+} \mathrm{IL}-6^{+} \mathrm{CD} 4^{+} \mathrm{T}$ cells are present in murine and human colon cancer tissues, are associated with tumour promotion and therefore may become a future therapeutic target limiting CRC progression.

majority of CRC appears to develop in a sporadic fashion. Interestingly, detection of a tumour-infiltrating lymphocyte (TIL) pool enriched for cytotoxic T cells is associated with a favourable prognosis of sporadic CRC cases compared with patients with reduced intratumoral T cell numbers. ${ }^{3}$ Collectively, mucosal inflammation seems to play a dual and contextdependent role exerting detrimental and beneficial effects during CRC development and progression.

Elevated interleukin (IL) 23/IL-17a expression levels within solid tumours negatively correlate with disease-free survival and overall survival in patients with CRC and other solid tumour entities. ${ }^{4}{ }^{5}$ The cellular origin of the IL-17a production within mucosal tumours is controversially discussed since a number of IL-17a-producing immune cell subsets including $\mathrm{CD}^{+} \alpha / \beta \mathrm{T}$ cells, so called Th17 cells, $\gamma \delta \mathrm{T}$ cells, innate lymphoid cells (ILCs) and neutrophils reside in the mucosal compartment. ${ }^{2}$ Naïve $\mathrm{CD}^{+} \mathrm{T}$ cells differentiate in response to a number of cytokines including IL-1ß, IL-6, IL-23 and TGF- $\beta$ into Th17 cells, a process which is critically dependent on the activating protein 1 family member basic leucine zipper transcription factor ATF-like (Batf). ${ }^{6}$ Beside its role during differentiation, IL-23 is crucial for Th17 cell maintenance and survival in vivo. ${ }^{7}$ However, whether IL-23 promotes Th17 cell biology in the colon and whether this interaction is essential for the establishment of an IL-23/IL-17a-enriched micromilieu linked to poor prognosis has not been thoroughly tested. In the present study we therefore sought to address the specific role of Batf-dependent signalling in Th17 cells and its consequences during colon cancer formation and progression.

\section{MATERIALS AND METHODS \\ Animals}

Mice were housed under pathogen-free conditions. For some experiments, male C57BL/6j and Balb/cJRj mice were purchased from Janvier (Le Genest-Saint-Isle, France). C57BL/6 Batf ${ }^{-1}$,

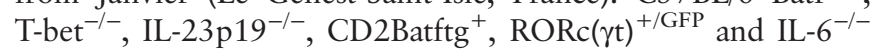
mice were previously described. ${ }^{6}{ }^{8-11}$ We thank the Wellcome Trust Sanger Institute Mouse Genetics Project (Sanger MGP) for providing the mutant mouse line $(\mathrm{B} 6 \mathrm{NTac} ; \mathrm{B} 6 \mathrm{~N}-\mathrm{A}<\mathrm{tm} 1 \mathrm{Brd}>$ Il23r $<$ tm2a(EUCOMM)Wtsi/Wtsi). ${ }^{12}$ Further materials and methods are available as an online supplementary file.

\section{RESULTS}

\section{Batf expression is increased together with IL-23p19 and IL-23R in human UC tissue}

IL-17a-driven immune responses were implied to be involved in the pathogenesis of immune-mediated colitis. ${ }^{2}{ }^{13} 14$ In line with previous reports we detected an increased frequency of IL-17a-expressing lamina propria (LP) cells during active colitis in IBD ex vivo (see table 1 and online supplementary figure S1) and elevated global IL-17a expression levels in UC and Crohn's disease (CD) tissue samples (figure $1 \mathrm{~A}$ ).
Table 1 Shift towards increased frequencies of colonic IL-17a ${ }^{+}$ versus IFN $\gamma^{+}$LP mononuclear cells (LPMCs) in IBD and CRC tissues

\begin{tabular}{llll}
\hline IBD & $\begin{array}{l}\% \text { IL-17a/ } \\
\text { IFN } \gamma^{+}\end{array}$ & CRC & $\begin{array}{l}\% \text { IL-17a/ } \\
\text { IFN } \gamma^{+}\end{array}$ \\
\hline Normal $(n=8)$ & $0.27(0.24)$ & Normal $(n=25)$ & $0.18(0.13)$ \\
UC $(n=8)$ & $0.34(0.28)$ & UICC I/II $(n=13)$ & $0.24(0.21)$ \\
CD $(n=8)$ & $0.34(0.30)$ & UICC III/IV $(n=10)$ & $0.32(0.28)$ \\
\hline
\end{tabular}

LPMCs were isolated from colon tissue of controls (normal), patients with IBD (UC and $C D$ ) and CRC of defined tumour stages in accordance with the classification of the UICC. For CRC analysis, normal tissue represents adjacent, tumour-free tissue of the same patient. Frequencies of IL-17a ${ }^{+}$and IFN $\gamma^{+} \mathrm{LP}$ cells were assessed by intracellular cytokine staining via flow cytometry as displayed in online supplementary figure $\mathrm{S} 1$ and ratios of IL-17a+ $/ \mathrm{FN} \gamma^{+} \mathrm{LP}$ cells were determined as indicated. Data show mean values with SD.

CD, Crohn's disease; CRC, colorectal cancer; LP, lamina propia; UICC, Union internationale contre le cancer; UC, ulcerative colitis.

Development of murine Th17 cells is critically dependent on the expression of the lineage-specifying transcription factors ROR $\gamma$ t and Batf. ${ }^{6}{ }^{15}$ However, the regulation of mucosal expression of Th17 associated transcription factors during immunemediated IBD has not been studied in detail. Interestingly, Batf expression levels were significantly increased in UC and to a lesser extent in $\mathrm{CD}$ samples in an inflammation-dependent manner (figure 1A). Moreover, Batf expression levels were strongly elevated within highly colitic tissue compared with macroscopically unaffected tissue of the same patients with UC while ROR $\gamma t$ expression levels were unaltered or even inversely reduced as colitis severity increased (see figure 1A and online supplementary figure S2). Importantly, elevated Batf levels within UC but not CD tissue samples were associated with significantly increased IL-23p19 and IL-23R expression. Together, our results demonstrate that unlike ROR $\gamma t$ Batf is highly expressed along with IL-23/IL-23R in UC but not in CD tissues.

\section{Batf but not RORyt is expressed within human CRC tissue}

CRC development and progression are accompanied by a shift towards an IL-23/IL-17a enriched microenvironment. ${ }^{4}{ }^{5}$ Intracellular cytokine expression analysis of TIL confirmed that CRC is associated with an increased frequency of IL- $17 \mathrm{a}^{+}$LP cells in a tumour-stage dependent manner compared with adjacent, tumour-free tissue (see table 1 and online supplementary figure S1). Accordingly, CRC tissue contained overall elevated IL-17a gene expression levels compared with control tissue (figure 1A). However, significantly increased IL-17a expression levels were largely restricted to advanced tumour stages suggesting that IL-17a expression might rather reflect tumour load than functional involvement in early tumorigenesis (see table 1 and online supplementary figures $\mathrm{S} 1$ and $\mathrm{S} 3$ ).

IL-23 and IL-6 are functionally related to IL-17a-producing immune cell subsets and represent common components of the tumour tissue-associated inflammatory network assumed to exert protumoral effects. ${ }^{4}{ }^{11} 1617$ Accordingly, we detected significantly elevated IL-23 and IL-6 expression levels within CRC samples compared with adjacent, tumour-free colon tissue while global IL-23R expression levels were unaltered (see figure $1 \mathrm{~A}$ and online supplementary figures S3 and S4). Interestingly IL-23 and IL-6 expression levels were increased in a CRC stageindependent manner compared with control tissue implying protumoral roles early in tumour development (see online supplementary figure S3 and S4).

Batf expression levels in CRC samples were significantly increased compared with control tissue in a CRC stage- 
A

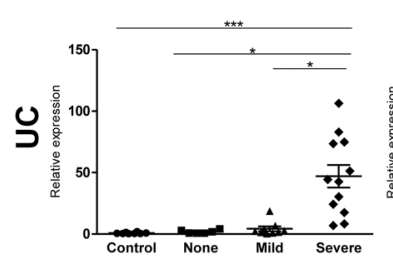

IL-23p19

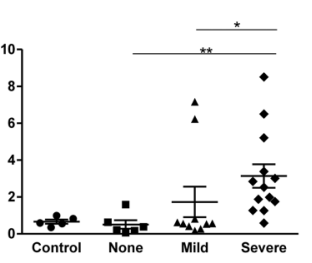

IL-23R

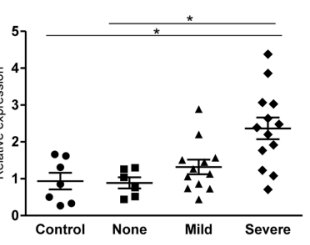

Batf
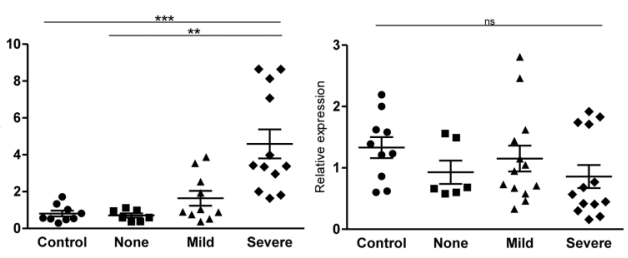
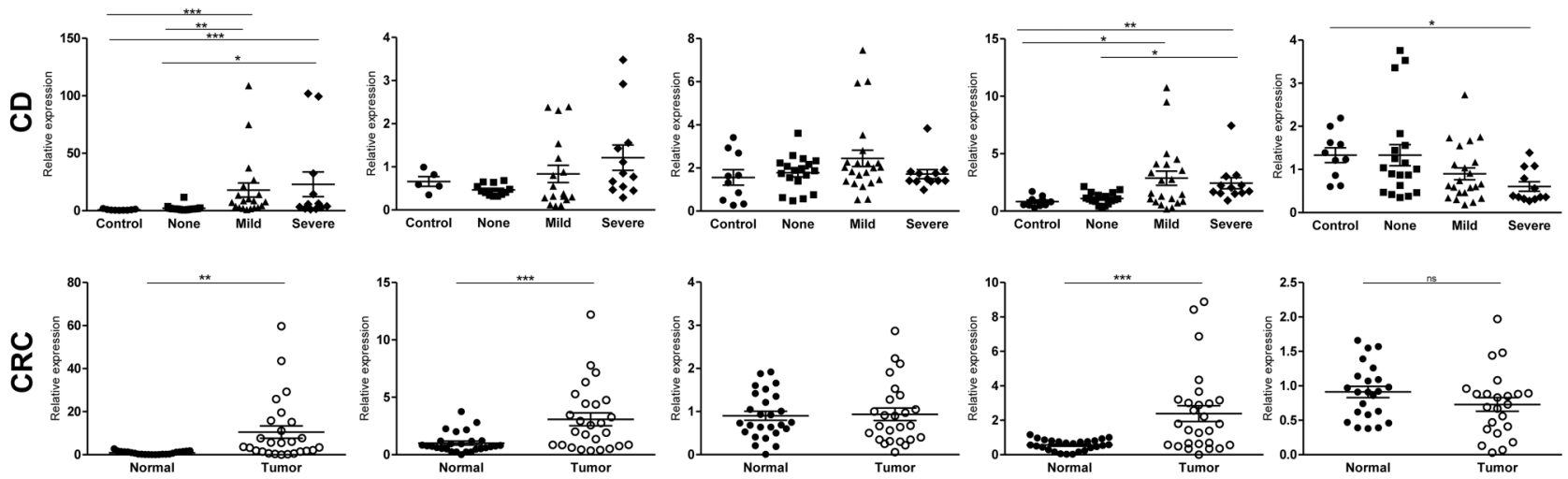

B
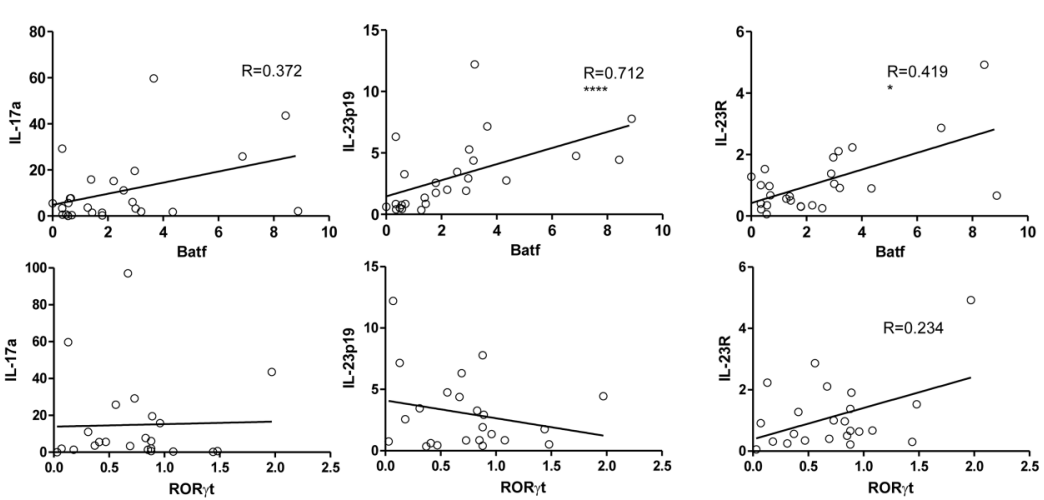

C
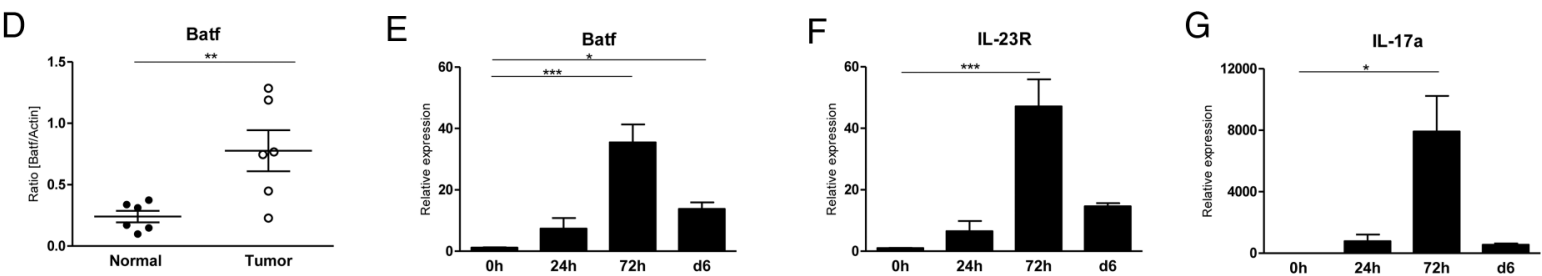

$\mathrm{H}$
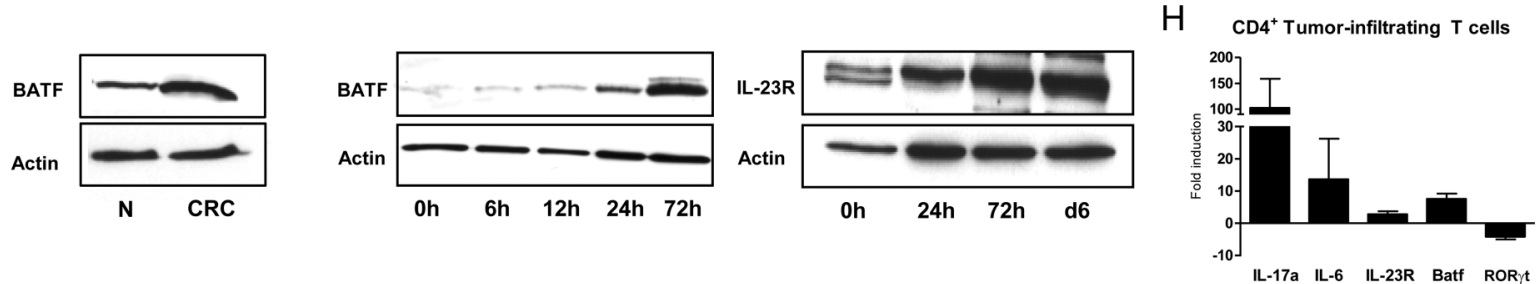

Figure 1 Human UC and colorectal cancer (CRC) contain a common Batf/IL-23p19/IL-23R tissue expression signature. (A) Colon tissue samples from healthy controls $(n=8)$, patients with UC $(n=30)$ (upper panel) or Crohn's disease $(C D)(n=50)$ (middle panel) colitis with indicated histological disease severities (none, mild, severe) and from patients with CRC $(n=27)$ using tumour and adjacent tumour-free tissue (normal) of the same patient with CRC (lower panel) were assessed for IL-17a, IL-23p19, IL-23R, Batf and RORyt mRNA expression by qPCR. (B) Correlation analysis of Batf (upper panel) and ROR $\gamma t$ (lower panel) expression, respectively, to IL-17a, IL-23p19 and IL-23R expression within CRC tumour samples. (C) Correlation analysis of Batf and ROR $\gamma$ t expression in CRC tumours. (D) Upper panel: Batf/actin protein expression ratios \pm SEM within normal and CRC tissues ( $n=6)$ assessed by densitometry. Lower panel: Batf protein detection via western blot normal $(\mathrm{N})$ and tumour tissues of the same patient with $\mathrm{CRC}$. Actin served as a loading control. (E-G) Batf, IL-23R and IL-17a gene and protein expression kinetics \pm SEM of either unstimulated (0 h) or IL-1B/LL-6/IL-23-stimulated human $C D 4^{+} T$ cells assayed by qPCR ( $E$, $F$ upper panels, $G$ ) or western blot ( $E$ and $F$, lower panels) representing at least three independent experiments. (H) Magnetically purified CD4 ${ }^{+}$tumour-infiltrating lamina propia (LP) T cells from three patients with CRC were stimulated for $6 \mathrm{~h}$ ex vivo with anti-CD3/anti-CD28. Indicated cytokine expression dynamics relative to mRNA expression in unstimulated control CD4 ${ }^{+}$LP T $_{\text {cells were assessed }}$ by $q$ PCR. Data represent mean values with \pm SEM. ${ }^{*} p<0.05 ;{ }^{* *} p<0.01 ;{ }^{* *} p<0.001 ;{ }^{* * * *} p<0.0001$. 
independent manner (see figure 1A, D and online supplementary figure S3). In striking contrast to Batf, ROR $\gamma t$ expression levels were unaltered in CRC versus tumour-free tissue. Interestingly, a similar induction of Batf but not ROR $\gamma t$ expression was detected within pancreatic adenocarcinoma samples compared with adjacent non-malignant pancreas tissue possibly implying a broader role for Batf-driven immune responses during adenocarcinoma formation (see online supplementary figure S5). In CRC tissue, Batf expression strongly correlated with IL-23 and to a lesser extent with IL-23R but not with IL-17a expression while ROR $\gamma \mathrm{t}$ expression lacked any correlation with IL-17a, IL-23 and IL-23R expression (see figure $1 \mathrm{~B}$ and online supplementary figure $\mathrm{S} 6$ ). Importantly across all tumour stages, intratumoral Batf and ROR $\gamma \mathrm{t}$ expression did not correlate either (see figure 1C, and online supplementary figure S6). Collectively, these data indicate that Batf expression is strongly associated with CRC formation and linked to intratumoral IL-23 and IL-23R but not IL-17a expression while ROR $\gamma \mathrm{t}$ expression is unaltered during CRC development.

\section{Human CRC tissue contain Batf ${ }^{+}$ROR $\gamma \mathrm{t}^{-} \mathrm{IL}^{-23 \mathrm{R}^{+} \mathrm{CD} 4^{+} \mathrm{T} \text { cells }}$ expressing IL-17a and IL-6}

Murine Th17 development is dependent on Batf. During human Th17 differentiation in vitro Batf expression was strongly upregulated together with Th17-associated IL-23R and IL-17a expression implying a similar fate-specifying role for Batf in human Th17 cells (figure $1 \mathrm{E}-\mathrm{G}$ ). To assess the in vivo characteristics of human $\mathrm{CD}^{+}{ }^{+}$TIL in CRC, we isolated CD4 ${ }^{+}$LP cells from tumour and control tissues, respectively, of patients with CRC, restimulated these $\mathrm{T}$ cells ex vivo and performed gene expression analyses. Compared with unstimulated controls, TIL $\mathrm{CD}^{+} \mathrm{T}$ cells expressed elevated IL-17a, IL-6 and IL-23R levels (figure 1H). Strikingly, CD4 ${ }^{+}$TIL expressed increased Batf levels while ROR $\gamma \mathrm{t}$ expression decreased. In contrast ROR $\gamma$ t was upregulated in $\mathrm{CD}^{-}$ TIL (see online supplementary figure S7). In summary, our data demonstrate that CRC tissue contains a related Batf/IL-23/IL-6 signature and is infiltrated by IL-23R ${ }^{+} \mathrm{IL}-6^{+} \mathrm{IL}-17 \mathrm{a}^{+} \mathrm{CD} 4^{+} \mathrm{T}$ cells expressing Batf but not ROR $\gamma$ t.

\section{Diminished tumour formation in $\mathrm{APC}^{\mathrm{min}} \mathrm{Batf}^{-1-}$ mice}

To assess the functional role of Batf, we used two murine models of colonic tumorigenesis. First, mice expressing a truncated version of the adenomatous polyposis coli (APC) gene product develop numerous intestinal and colonic tumours (multiple intestinal neoplasia, $\left.\mathrm{APC}^{\mathrm{min}}\right) .{ }^{18}$ Sixteen-week-old $\mathrm{APC}^{\mathrm{min}} \mathrm{Batf}^{-/-}$mice developed less and smaller intestinal tumours compared with matched $\mathrm{APC}^{\text {min }} \mathrm{Batf}^{+/+}$mice while colon tumour formation was not unaffected (see online supplementary figure S8A,B). Interestingly, diminished tumour formation occurred despite unaltered intratumoral IL-17a expression (see online supplementary figure S8C). Together, these results suggest a protumoral, but IL-17a-independent role of Batf during sporadic intestinal tumorigenesis.

\section{Reduced CAC development in Batf ${ }^{-1-}$ mice}

Colitis-associated cancer (CAC) formation can be mimicked by treating mice with the carcinogen azoxymethan (AOM) intraperitoneally followed by cycles of dextran sodium sulfate (DSS)-induced colitis resulting in clinical colon tumour manifestation about 6 weeks after treatment initiation. ${ }^{19}$ Interestingly, CAC tissue expressed significantly increased Batf and IL-17a but not ROR $\gamma$ t levels compared with tumour-free colon tissue (figure 2A) mirroring elevated Batf and unaltered ROR $\gamma \mathrm{t}$ expression in human CRC samples.
To assess the role of Batf during CAC development, AOM/ DSS-treated Batf ${ }^{+/+}$and Batf ${ }^{-/-}$mice were monitored for colon tumour development. Expectedly, Batf ${ }^{+/+}$mice developed polyp-shaped tumours located predominately in the distal and middle parts of the colon (figure 2B-E). Narrow-band imaging analysis showed an altered vascularisation pattern confirming the neoplastic nature of these lesions (figure 2C). In contrast, Batf $^{-/-}$mice displayed reduced polypoid tumour formation in number and size resulting in a significantly diminished overall polypoid tumour load (figure $2 \mathrm{~B}-\mathrm{E}$ ). In line with the clinical appearance, histopathological studies showed that $\mathrm{Batf}^{+/+}$ tumours uniformly contained areas with high-grade dysplasia and predominantly polypoid (about 75\%)-rather than flatshaped (about 25\%) - carcinomas while only about $40 \%$ of the Batf $^{-/-}$tumours diminished in size and number included dysplastic and predominately flat appearing carcinomatous lesions (figure 2F). The inflammatory infiltrates within CAC-associated macroscopic tumours were indistinguishable between Batf $^{+/+}$ and $\mathrm{Batf}^{-/-}$mice (figure 2D, F).

\section{CAC development is critically regulated by Batf expressing $\mathrm{T}$ lymphocytes}

To test whether lymphocytes are required for the reduced tumour manifestation in the absence of Batf, AOM/DSS-induced tumour development was assessed on the $\operatorname{Rag} 1^{-/}$background. Importantly, irrespective of Batf expression macroscopic tumour burden was comparable between both $\operatorname{Rag} 1^{-/-}$mouse lines (figure 3A). Histological evaluations did not reveal differences in the epithelial morphology, pattern of dysplastic areas or the colitis activity (figure 3B, C). Accordingly, comparable numbers of colon-infiltrating myeloid peroxidase granulocytes were detected in both groups (figure 3D).

To investigate the $\mathrm{T}$ cell-intrinsic role of Batf for CAC development, we took advantage of CD2Batf transgenic $\left(\mathrm{CD} 2 \mathrm{Batftg}^{+}\right)$mice displaying increased Th17 cell differentiation potential in vitro and in vivo due to forced $\mathrm{T}$ cell-intrinsic Batf expression. ${ }^{6}$ Using this model system, we found an increased tumour load in $\mathrm{CD} 2 \mathrm{Batftg}^{+}$compared with nontransgenic control mice (figure $3 \mathrm{E}$ ). Together, reduced CAC formation in $\mathrm{Batf}^{-/-}$mice strictly depends on the presence of lymphocytes. Furthermore, results from the CD2Batftg ${ }^{+}$mouse model suggest that in fact Batf-dependent Th17 cells are causally promoting CAC development.

\section{Regulation of CAC development by Batf expressing T cells is independent of IL-17a expression}

To characterise the cytokine profile operative in tumourinfiltrating $\mathrm{T}$ cells, colonic LP $\mathrm{T}$ cells from naïve or $\mathrm{AOM} /$ DSS-challenged Batf ${ }^{+/+}$and Batf ${ }^{-/-}$mice or CD2Batftg ${ }^{+}$and $\mathrm{CD}_{2} \mathrm{Batftg}^{-}$mice, respectively, were analysed by intracellular cytokine staining. The LP fraction of AOM/DSS-treated tumourbearing Batf $^{+/+}$mice contained a significantly increased fraction of $\mathrm{CD}^{+}$Th17 cells compared with untreated mice while IFN $\gamma^{+} \mathrm{CD} 4^{+} \mathrm{T}$ cells were more frequent in $\mathrm{Batf}^{-/-}$LP preparations (figure 4A). Importantly, CAC formation and presence of tumour-associated Th17 cells were dependent on T cell-intrinsic Batf expression since AOM/DSS-challenged tumour-bearing $\mathrm{CD}_{2} \mathrm{Batftg}^{+}$mice contained elevated numbers and $\mathrm{Batf}^{-1-}$ mice diminished numbers of mucosa-infiltrating IL- $17 \mathrm{a}^{+} \mathrm{CD} 4^{+} \mathrm{T}$ cells compared with controls (see figure 4A and online supplementary figure S9). Strikingly, global IL-17a gene expression within Batf $^{-/-}$AOM/DSS-treated colon tissue significantly exceeded levels detected in $\mathrm{Batf}^{+/+}$tumours (figure 4B). Furthermore, Batf $^{-/-}$LP cells produced substantial amounts of IL-17a protein 
A
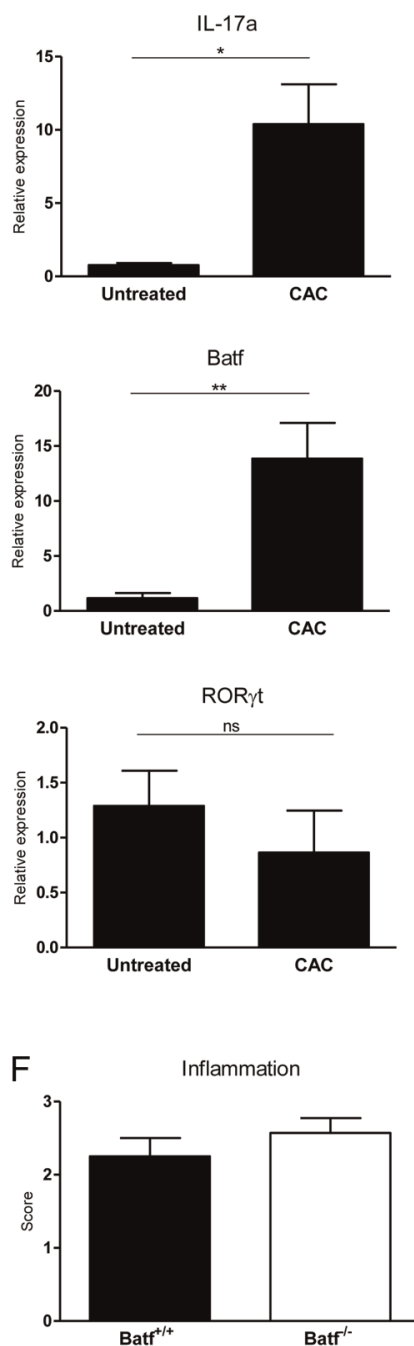

B
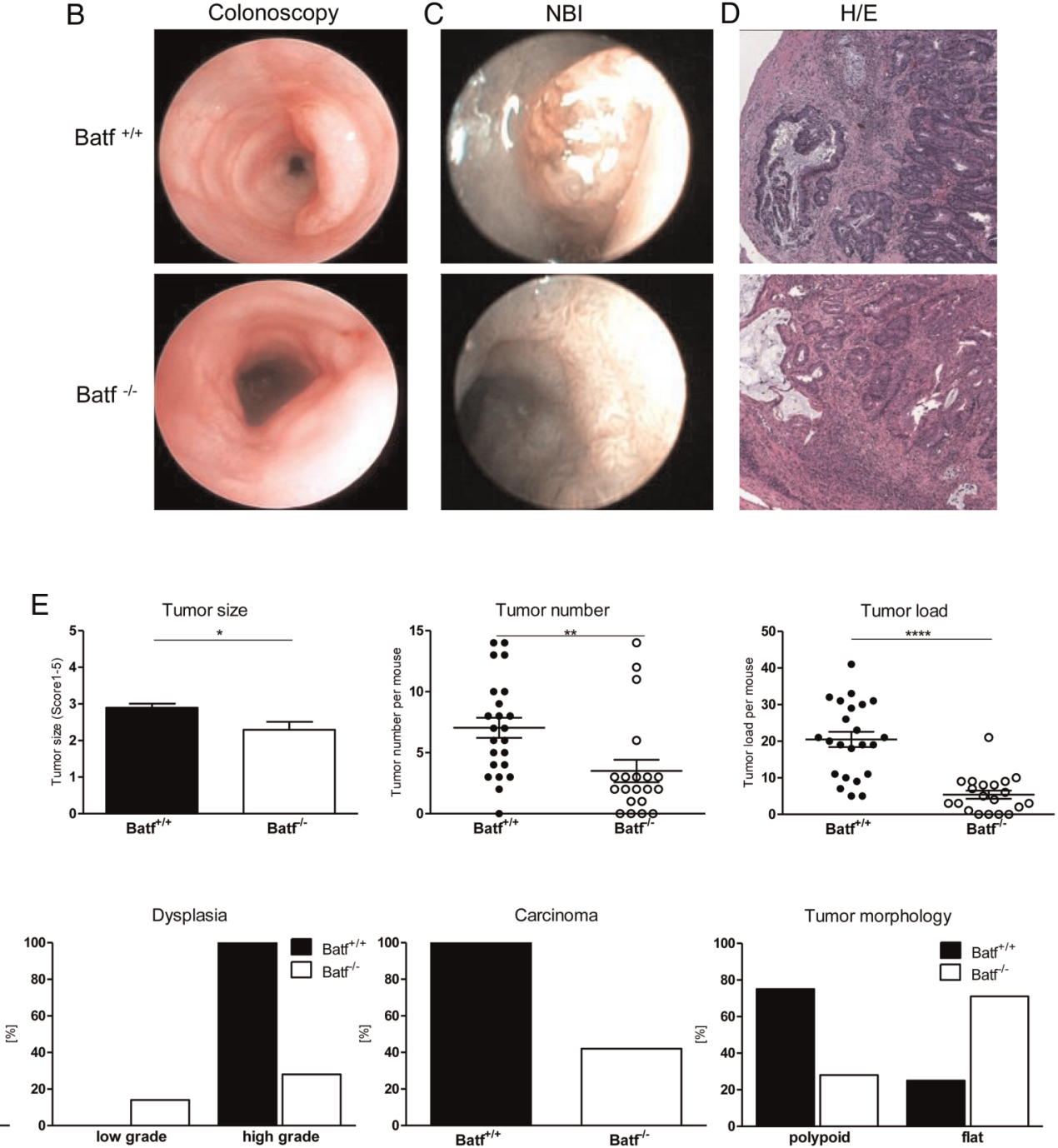

Figure 2 Inflammation-induced colon tumour development is reduced in the absence of Batf. Azoxymethan/dextran sodium sulfate (AOM/ DSS)-treated Batf ${ }^{+/+}$and Batf ${ }^{-1}$ mice were sacrificed on day 80. (A) Quantification of Batf, IL-17a and RORyt colonic expression levels in untreated compared with tumour-bearing Batf ${ }^{++}$mice $(n=18)$ by qPCR. (B) Representative endoscopic, (C) Narrow-band imaging (NBI) images and (D) representative H\&E images displaying polypoid tumours $(100 \times)$ of the distal colon. (E) Size, number and load of polypoid tumours of Batf ${ }^{++}$and Batf $^{-{ }^{-}}$mice ( $n=20 /$ genotype). (F) Macroscopically polypoid appearing colitis-associated cancer (CAC) tissue $(n=4-7 /$ genotype) was removed and assessed for signs of inflammation \pm SEM, dysplasia, carcinoma and tumour morphology (shown in \% presence/ all mice). Data represent mean values \pm SEM. ${ }^{*} \mathrm{p}<0.05 ;{ }^{* *} \mathrm{p}<0.01 ;{ }^{* * * *} \mathrm{p}<0.0001$.

comparable to $\mathrm{Batf}^{+/+}$controls after $\mathrm{T}$ cell receptor (TCR) stimulation ex vivo (figure 4B). Therefore we assessed whether Batf expression is required for the development and functionality of IL-17a producing TCR $\gamma / \delta^{+}$T cells and ILCs. ${ }^{8}{ }^{20}$ First, TCR $\gamma / \delta^{+}$T cell frequencies within the indicated lymphoid and non-lymphoid tissues were indistinguishable between $\mathrm{Batf}^{+/+}$ and $\mathrm{Batf}^{-/-}$mice (see online supplementary figure S10). The functionality of $\mathrm{Batf}^{--} \mathrm{TCR} \gamma / \delta^{+} \mathrm{T}$ cells was uncompromised since lymphoid-resident and non-lymphoid-resident $\mathrm{Batf}^{-1}$ ${ }^{-} \mathrm{TCR} \gamma / \delta^{+}$T cells secreted IL-17a levels comparable to Batf ${ }^{+/+}$ controls while $\mathrm{Batf}^{--} \mathrm{TCR} \alpha / \mathrm{B}^{+} \mathrm{T}$ cells expectedly produced reduced IL-17a protein levels (figure 4C).

Furthermore, ILC subset development was unaltered in $\mathrm{Batf}^{-/-}$ mice as assessed by flow cytometric analyses of ROR $\gamma t$ reporter gene and ILC-related surface marker (IL-7R, c-kit, CD4 and NKp46) expression (see online supplementary figure S11). ${ }^{8}$ Third, frequencies of IL-17a ${ }^{+}$and IL-22+ $2^{+}$ILCs within the LP compartment (see online supplementary figure $12 \mathrm{~A}-\mathrm{C}$ ), colonic
IL-17a and IL-22 expression (see online supplementary figure S12D) and overall IL-17a and IL-22 production by LP CD4 ${ }^{+}$ and $\mathrm{CD}^{-}$ILCs of $\mathrm{AOM} / \mathrm{DSS}$-treated $\mathrm{Rag} 1^{-/} \mathrm{Batf}^{+/+}$and $\operatorname{Rag} 1^{-/} \mathrm{Batf}^{-/-}$mice were indistinguishable (figure 4D). Finally, neutrophils produced IL-17a and IL-22 in a Batf-independent manner (figure 4E). Collectively, development and functionality of TCR $\gamma / \delta^{+} \mathrm{T}$ cells, ROR $\gamma \mathrm{t}^{+}$ILCs and neutrophils are unaltered in $\mathrm{Batf}^{-/-}$mice leading to indistinguishable expression levels of IL-17a and IL-22 in colon tissue despite Th17 deficiency. These results support the concept that Batf-dependent Th17 cells regulate CAC formation in an IL-17a independent manner.

\section{Reduced CAC formation in Batf $^{-1-}$ mice is not dependent on T-bet ${ }^{+}$IFN $\gamma^{+}$LP T cells}

To exclude the possibility that altered mucosal tumour development is due to the relative abundance of mucosal IFN $\gamma^{+}$Th1 $\mathrm{CD} 4^{+} \mathrm{T}$ cells present in the LP compartment of AOM/DSS-treated Batf $^{-/}$mice (figure 4A), we generated T-bet ${ }^{-/} \mathrm{Batf}^{-/-}$mice. The 


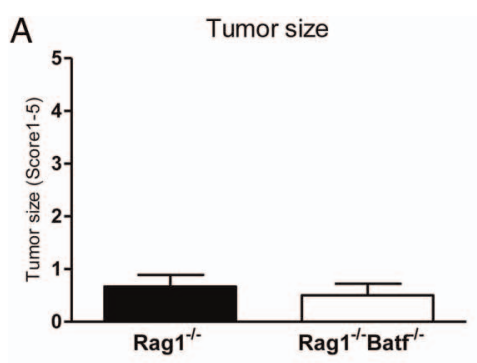

B

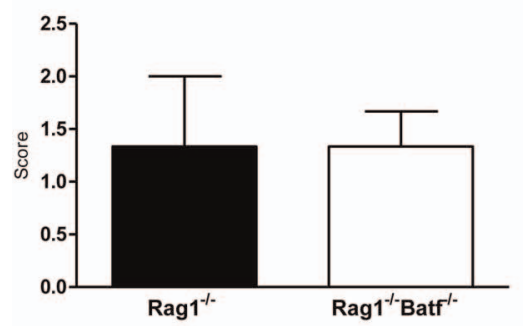

Rag $1^{-1}$

C

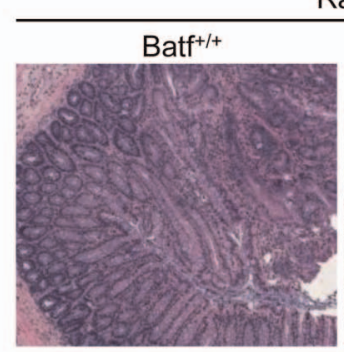

$\mathrm{E}$

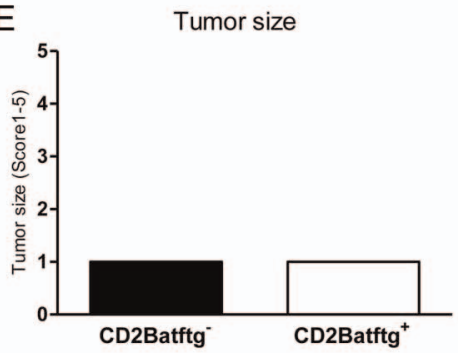

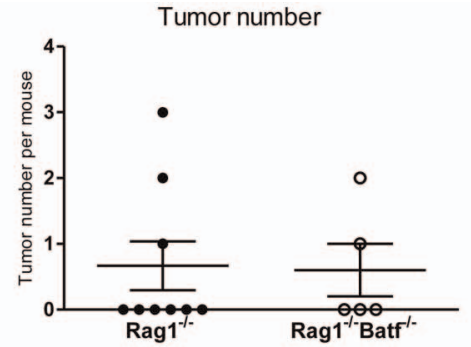

Epithelial morphology

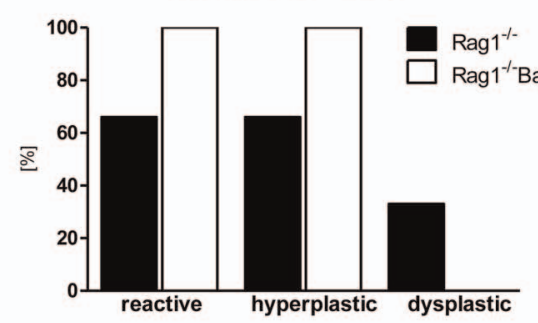

Tumor load

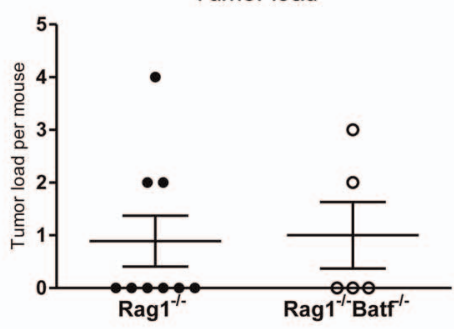

Dysplasia

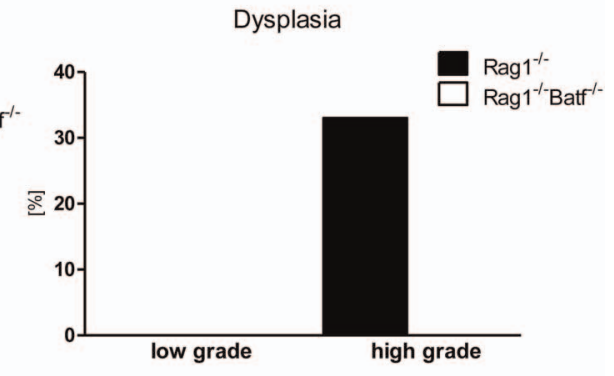

Rag $1^{-1}$
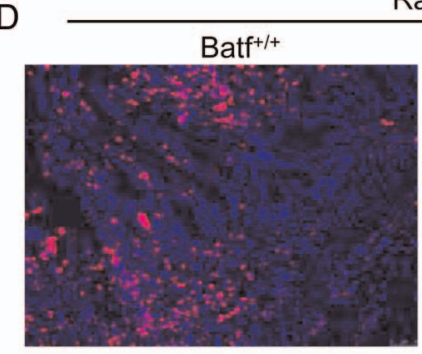

Batf $-1-$
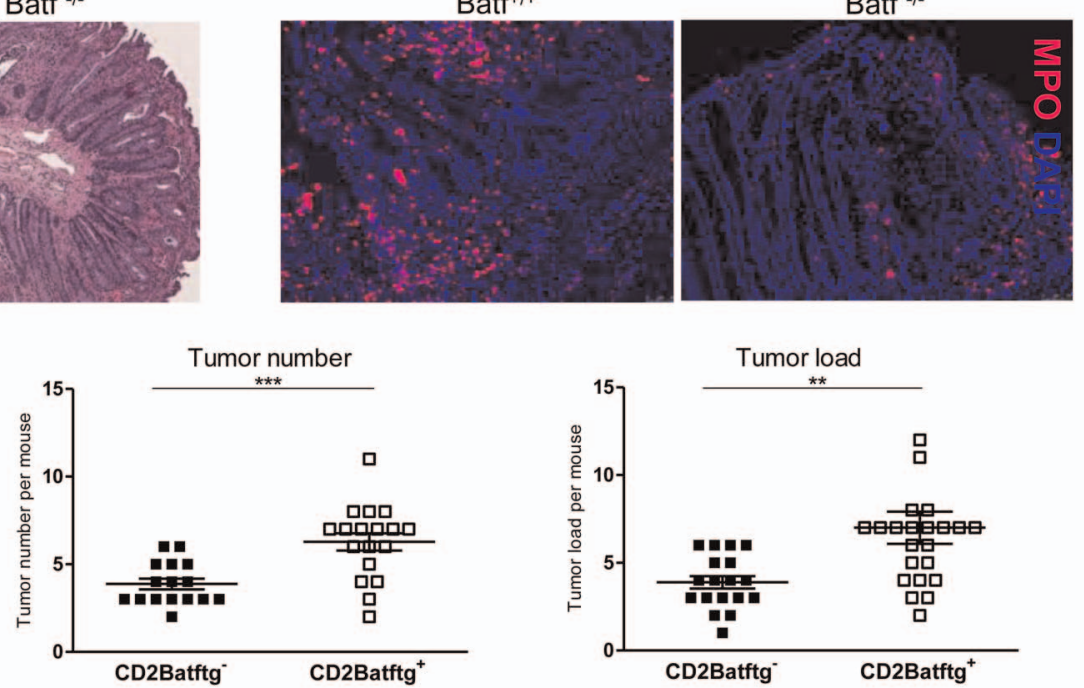

Figure 3 Batf expression within T cells promotes colitis-associated colon tumour formation. Rag ${ }^{-1-}$ Batf $^{+/+}$and Rag $1^{-l-}$ Batf $^{-1-}$ mice were treated with azoxymethan/dextran sodium sulfate (AOM/DSS) and sacrificed on day 94. (A) Clinical assessment of size, number and load of polypoid tumours \pm SEM ( $n=5-9 /$ genotype). (B) Histopathological assessment of epithelial morphology, dysplasia (shown in \% presence/all mice) and tissue inflammation \pm SEM. (C) Representative H\&E stained distal colon sections (100x) and (D) immunofluorescence myeloperoxidase (MPO, red) staining with nuclei counterstaining (Hoechst, blue). (E) AOM/DSS-treated CD2Batftg ${ }^{+}$and CD2Batftg- mice were clinically assessed for size, number and load of polypoid tumours (from left to right) on day 80 . Data show mean values \pm SEM. ${ }^{* *} \mathrm{p}<0.01 ;{ }^{* *} \mathrm{p}<0.001$.

transcription factor T-bet is a critical transcriptional regulator controlling Th1 development. ${ }^{9}$ Expectedly, IFN $\gamma^{+} \mathrm{CD}^{+}{ }^{+} \mathrm{T}$ cell frequencies in spleen, $\mathrm{mLN}$ and LP were significantly reduced in AOM/DSS-treated $\mathrm{T}^{-}$bet $^{-/}$Batf $^{-/}$mice compared with $\mathrm{T}^{-}$bet $^{+/}$ ${ }^{+} \mathrm{Batf}^{+/+}$and $\mathrm{T}-\mathrm{bet}^{+/+} \mathrm{Batf}^{-/-}$mice (figure 5A). However, additional T-bet deficiency of $\mathrm{Batf}^{-/-}$mice did not reconstitute CAC formation excluding that reduced CAC development in the absence of Batf-dependent Th17 cells is resulting from an increased frequency of IFN $\gamma^{+} \mathrm{CD}^{+}{ }^{+} \mathrm{LP}$ T cells (figure 5B, C).

\section{IL-23p19/LL-23R expression in AOM/DSS-induced CAC tissue is regulated by Batf-expressing T cells}

Similarly to our results in human UC and CRC samples, we detected a strong induction of IL-23 expression within CAC samples derived from AOM/DSS-treated $\mathrm{Batf}^{+/+}$mice compared with healthy control colon tissue (figure $6 \mathrm{~A}$ ). In contrast, Batf ${ }^{-/}$ CAC tissue contained, despite unaltered IL-17a expression levels, reduced IL-23 expression levels compared with colon tissue of tumour-bearing $\mathrm{Batf}^{+/+}$mice. Albeit lacking significance, this trend was also observed on the protein level (figure 6A, B). Interestingly, IL-23p19 expression differences were abrogated in the absence of lymphocytes (see online supplementary figure S12D). Furthermore, inflammation-induced, but not sporadic colon tumour formation was associated with reduced intratumoral IL-23R gene expression in $\mathrm{Batf}^{-/-}$compared with Batf ${ }^{+/+}$ mice (see figure 6D and online supplementary figure $8 \mathrm{C}$ ). Conversely, tumours from CD2Batftg ${ }^{+}$mice expressed elevated IL-23p19 and IL-23R transcript levels compared with nontransgenic controls (figure 6C, D). Reduced colon tumour burden and diminished intratumoral IL-17a expression levels in 
A

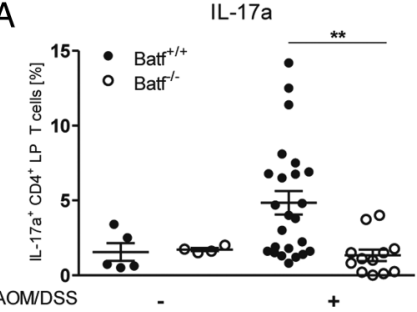

C

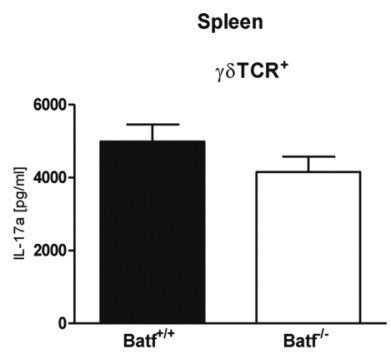

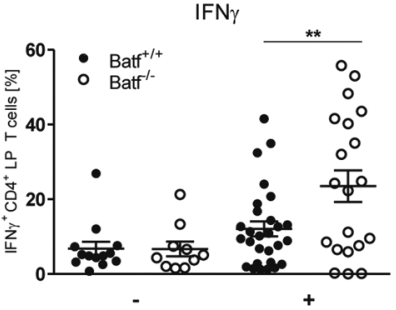

Small intestine

$\gamma \delta \mathrm{TCR}^{+}$

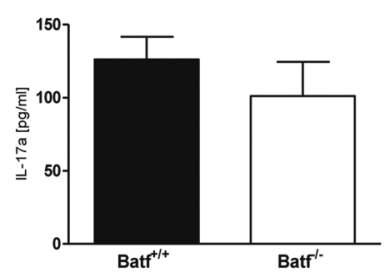

B

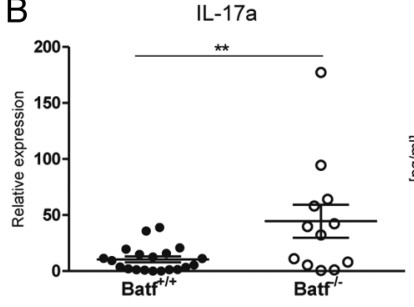

IL-17a

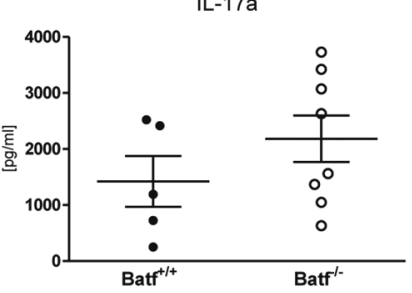

D

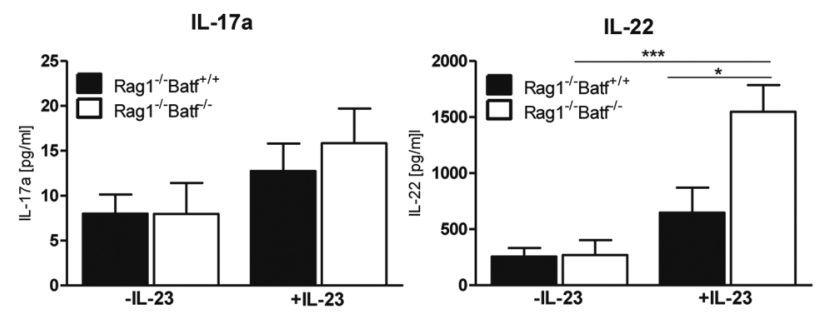

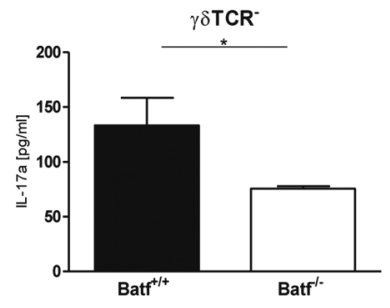

E

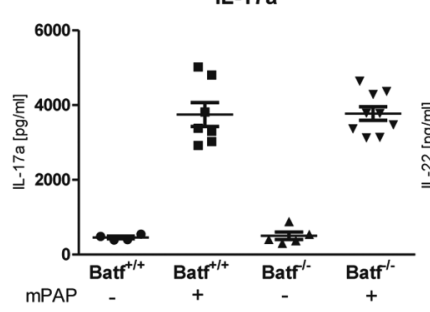

mPAP
$\mathrm{Ly}^{6} \mathrm{G}^{+} \mathrm{CD} 11 \mathrm{~b}+\mathrm{BM}$

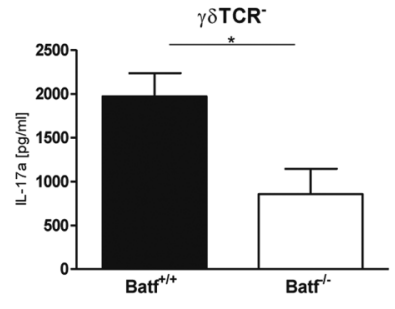

Batf

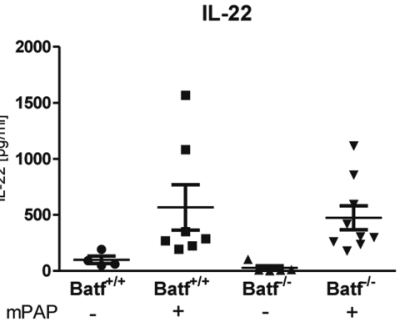

Figure 4 Reduced colitis-associated cancer development in Batf ${ }^{-1-}$ mice is independent of IL-17a/IL-22. Azoxymethan/dextran sodium sulfate (AOM/DSS)-treated Batf ${ }^{+/+}$and Batf ${ }^{-\prime-}$ mice were sacrificed on day 80. (A) Frequency of IL-17a $\mathrm{a}^{+}$and IFN $\gamma^{+} \mathrm{CD} 4^{+}$colonic lamina propia (LP) T cells of untreated or AOM/DSS-treated Batf ${ }^{+/+}$and Batf $^{-l-}$ mice assessed by intracellular cytokine staining ex vivo. (B) Quantification of IL-17a expression in AOM/DSS-treated colon tissue by qPCR (left) and IL-17a protein levels in LP cell culture supernatants after LP cells from AOM/DSS-treated Batf ${ }^{+/+}$ and Batf $^{-l-}$ mice were stimulated again with anti-CD3/anti-CD28 ex vivo (right) by ELISA. (C) $\gamma \delta \mathrm{TCR}^{+}$(top) or $\gamma \delta \mathrm{TCR}^{-}$cells (bottom) were purified from indicated organs and ex vivo challenged with anti-CD3/anti-CD28 and IL-23 for $24 \mathrm{~h}$. Cell culture supernatants were assayed for IL-17a with ELISA. (D) Rag1 ${ }^{-l-}$ Batf $^{+/+}$and Rag1 ${ }^{-I-}$ Batf $^{-I-}$ mice were treated with AOM/DSS and sacrificed on day 94. Culture supernatants of untreated (-IL-23) or for $24 \mathrm{~h}$ with IL-23 (200 ng/mL) stimulated LP cells were collected and assayed for IL-17 and IL-22 levels with ELISA. (E) Magnetically purified bone marrow derived Ly6G $\mathrm{G}^{+} \mathrm{CD} 11 \mathrm{~b}^{+}$neutrophils from Batf ${ }^{+/+}(\mathrm{n}=8)$ and Batf ${ }^{-I}(\mathrm{n}=8)$ mice were cultured for $3 \mathrm{~h}$ in Dulbecco's modified eagle's medium DMEM before cells were left untreated (-) or stimulated (+) with $200 \mu \mathrm{g} / \mathrm{ml}$ soluble murine peroxidase-anti-peroxidase immune complexes (mPAP) for $1 \mathrm{~h}$. Protein concentrations for IL-17a and IL-22 were measured in the cell-free supernatant using ELISA. Data show mean values of three independent experiments \pm SEM. ${ }^{*} p<0.05 ;{ }^{* *} p<0.01 ;{ }^{* *} p<0.001$. TCR, T cell receptor.

IL-23p $19^{-/-}$compared with IL-23p $19^{+/+}$mice provided functional proof for the crucial role of IL-23-driven pathogenesis of inflammation-induced colon tumour formation (figure $6 \mathrm{E}-\mathrm{F}$ ). Together, results from human and murine studies indicate that IL-23/IL-23R and Batf expression levels within colon tumour tissue are interdependent implying a Batf-dependent but IL-17a-independent regulation of intratumoral IL-23/IL-23R expression levels via Th17 cells.

\section{Batf governs the generation of IL-23 induced IL-23R ${ }^{+}$Th17 cells}

Batf is indispensable for TGF-ß/IL-6-induced Th17 polarisation. ${ }^{6}$ Recently, a TGF-ß-independent, IL-1ß/IL-6/IL-23-induced Th17 subpopulation-so-called IL-23(Th17) cells—was described as a crucial participant during Th17-driven tissue inflammation in vivo. ${ }^{7}$ IL-23(Th17) polarisation experiments of naïve $\mathrm{CD}^{+} \mathrm{T}$ cells revealed that Batf deficiency resulted in a strong reduction of IL-17a gene and protein expression compared with Batf $^{+/+} \mathrm{T}$ cells (figure $7 \mathrm{~A}$ ). Interestingly, ROR $\gamma \mathrm{t}$ expression was abrogated in IL-23(Th17) polarised Batf $^{-1-}$ $\mathrm{T}$ cells while Batf expression was largely maintained in the absence of ROR $\gamma$ t (see online supplementary figure S13A). Conversely, forced transgenic expression of Batf significantly induced ROR $\gamma \mathrm{t}$ expression along with IL-17a gene and protein expression compared with non-transgenic T cells (see figure 7B and online supplementary figure S13B). Together IL-23(Th17) cell development depends on Batf acting upstream of ROR $\gamma \mathrm{t}$ as Batf regulates ROR $\gamma$ t expression while the regulation of Batf expression appears to be ROR $\gamma \mathrm{t}$-independent.

IL-23 is produced by antigen-presenting cells (APCs) and was shown to promote development, maintenance and expansion of IL-23R ${ }^{+}$cells including Th17 cells in vivo. ${ }^{7}$ During murine and human Th17 differentiation IL-23R expression is progressively upregulated rendering Th17 cells increasingly responsive to IL-23 signals (figures $1 \mathrm{~F}$ and 7 ). Previous studies suggested that IL-6/TGF-ß3-induced IL-23R expression on $\mathrm{T}$ cells is directly regulated by Batf. $^{6}{ }^{15}$ Under IL-23(Th17) polarisation conditions Batf ${ }^{+/+}$but not Batf $^{-/-}$T cells upregulated IL-23R expression (figure 7A, left column). To model repetitive $\mathrm{T}$ cell stimulation conditions as anticipated during CAC formation, we cultured naïve Batf $^{+/+}$and Batf ${ }^{-/-}$CD4 ${ }^{+}$T cells under IL-23 (Th17) conditions for 5 days. After 2 days of rest in the 


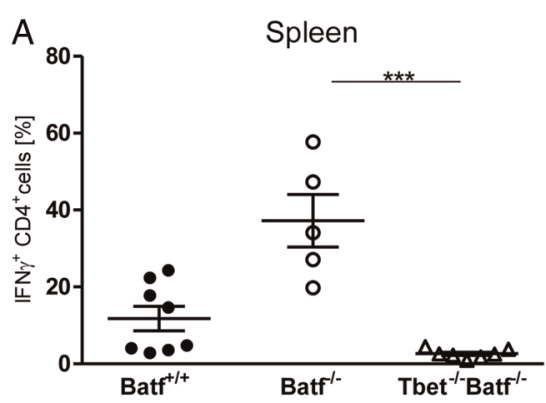

B

Batf $^{+/+}$
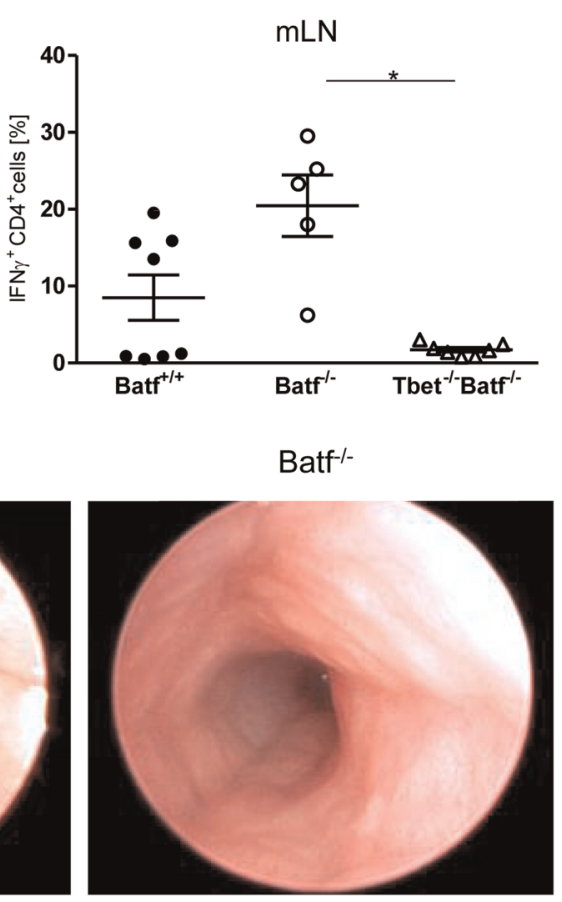

C

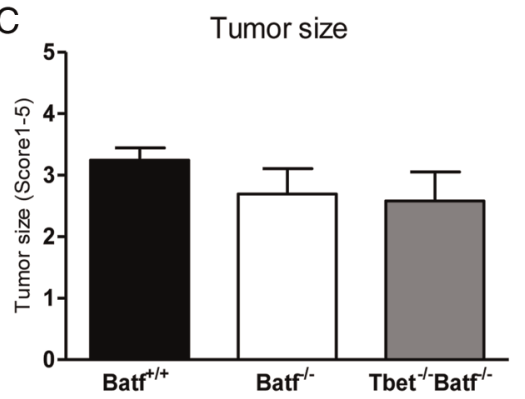

Batf $^{--}$

Tumor number
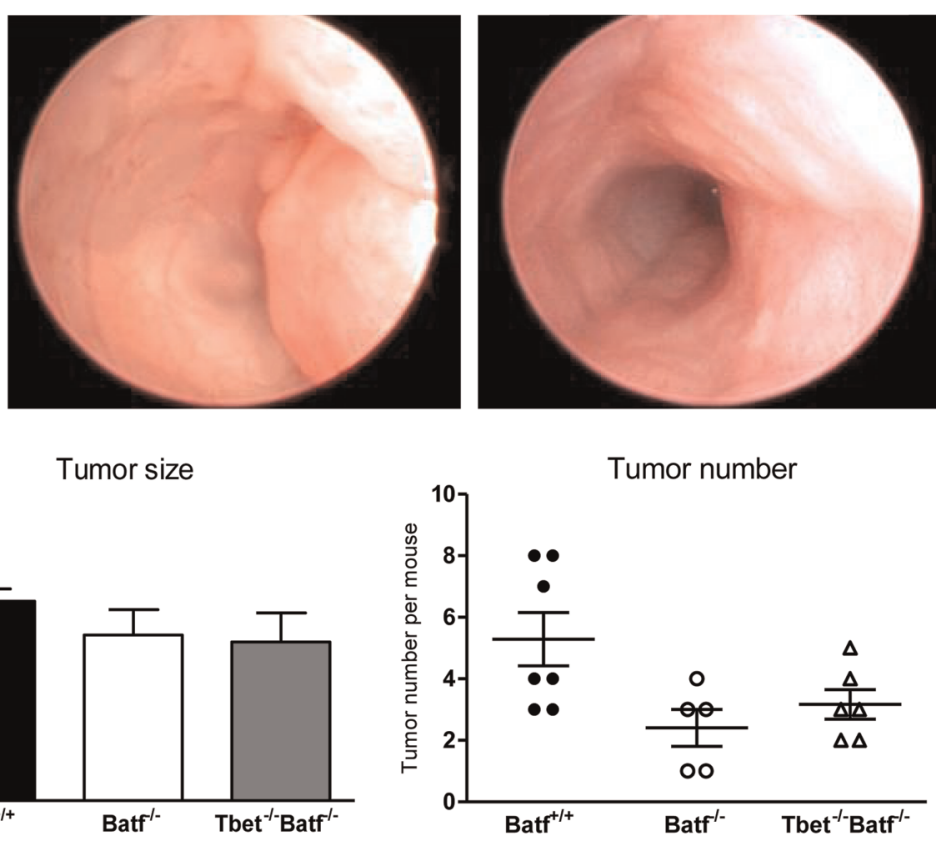

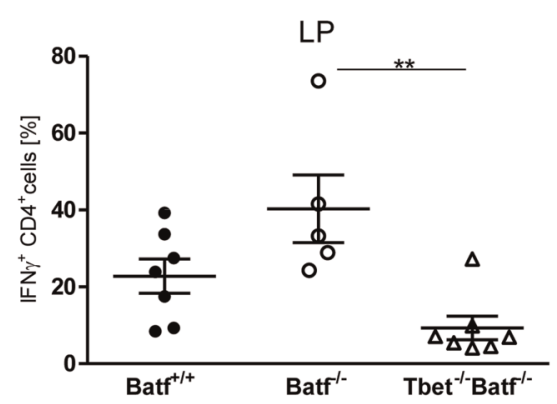

Tbet $^{-/}$Batf ${ }^{-/}$

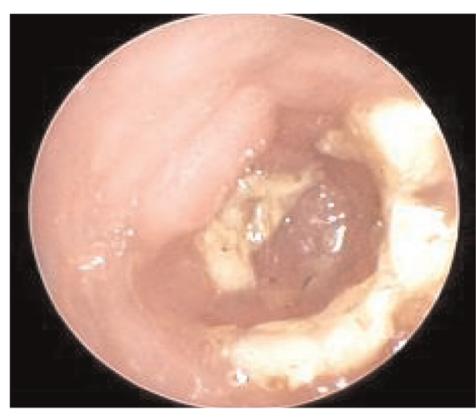

Tumor load

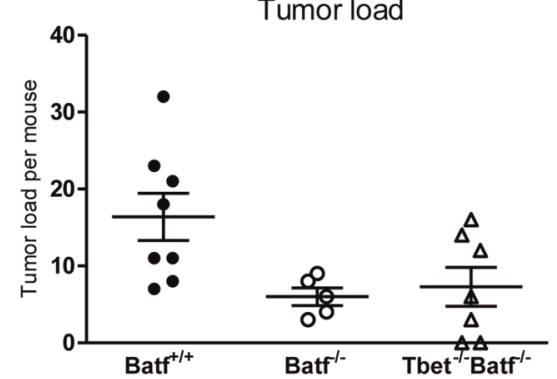

Figure 5 Batf ${ }^{-I-}$ IFN $\gamma^{+}$Th1 cells are not required for diminished colon tumour formation. Batf ${ }^{+/+}$, Batf ${ }^{-1-}$ and T-bet ${ }^{-1-}$ Batf $^{-1-}$ mice were treated with azoxymethan/dextran sodium sulfate (AOM/ DSS) and sacrificed on day 90. (A) Frequency of IFN $\gamma^{+} \mathrm{CD} 4^{+}$colonic lamina propia (LP) T cells of AOM/DSS-treated Batf ${ }^{+/+}$, Batf $^{-/-}$and Tbet $^{-/-}$Batf $^{-/-}$mice assessed by flow cytometry for spleen, mesenteric lymph node (mLN) and LP. (B) Representative endoscopic images of the distal colon. (C) Size, number and load of colitis-associated cancer tumours of Batf ${ }^{+/+}$, Batf ${ }^{-I-}$, T-bet $^{-\prime}$ ${ }^{-}$Batf $^{-I-}$ mice $(n=7)$. Data represent mean values \pm SEM. ${ }^{*} p<0.05 ;{ }^{* *} p<0.01 ;{ }^{* * *} p<0.001$.

presence of IL-2, IL-23(Th17) cells were again stimulated in the presence of IL-23 and analysed on day 9. Similarly to day 5, IL-17a production and IL-23R expression by IL-23(Th17) cells stimulated again were mainly Batf-dependent (figure 7A, right column). In line with a central regulatory role of Batf during IL-23R expression, CD2Batftg ${ }^{+} \mathrm{T}_{\text {cells }}$ expressed elevated levels of IL-23R compared with non-transgenic $\mathrm{T}$ cells (figure $7 \mathrm{~B}$ ). IL-23R expression and consecutive IL-23 responsiveness of Th17 cells are functionally crucial however since IL-23R ${ }^{-/-} \mathrm{T}$ cells expressed reduced IL-17a transcript and protein levels indicating hampered Th17 development (figure 7C). Together, IL-23R expression by murine Th17 cells is Batf-dependent while IL-23R-mediated signals are critical for proinflammatory Th17 cell differentiation in vitro.

\section{Tumour-infiltrating CD4 ${ }^{+}$LP T cells are IL-23R ${ }^{+}$and express a unique Batf-dependent expression profile}

Batf $^{-/-}$sporadic and CAC tumours-although reduced in size and number-contained IL-6 levels comparable to Batf $\mathrm{B}^{+/+}$ tumours (see online supplementary figure S8C and figure 8). While in vitro differentiated $\mathrm{CD}^{+}{ }^{+} \mathrm{T}$ cells do not produce IL-6 irrespective of (IL-23)Th17 conditions or Batf expression (see online supplementary figure S14), ${ }^{21}$ gene expression analysis of sort-purified $\mathrm{CD}^{+}$and $\mathrm{CD}^{-}$LP $\mathrm{T}$ cells from $\mathrm{AOM} /$ DSS-treated Batf ${ }^{+/+}$mice ex vivo confirmed substantial IL-6 expression by both subsets. In contrast we were unable to detect IL-6 expression by Batf $^{-/-}$CD ${ }^{+}$T cells while IL-6 expression by $\mathrm{CD}^{-} \mathrm{LP}$ mononuclear cells was uncompromised (see figure $8 \mathrm{~B}$ and online supplementary figure S15B). Importantly, in line with our in vitro studies ex vivo analysis of Batf ${ }^{-/-} \mathrm{CD}^{+}{ }^{+} \mathrm{LP} \mathrm{T}$ cells confirmed diminished IL-17a and IL-23R expression levels in vivo (figure $8 \mathrm{~B}$ ) while IFN $\gamma$ expression was increased compared with $\mathrm{Batf}^{+/+}$control cells (see online supplementary figure $15 \mathrm{~A}$ and figure $4 \mathrm{~A}$ ). Collectively, our data suggest that the presence of intratumoral IL- $17 \mathrm{a}^{+} \mathrm{IL}-23 \mathrm{R}^{+} \mathrm{IL}-6^{+} \mathrm{CD} 4^{+} \mathrm{LP}$ T cells is Batf-dependent and associated with CAC development.

\section{IL-6/IL-6R administration largely reconstitutes CAC formation in Batf $^{-1-}$ mice}

IL-6 mediated effects on intestinal epithelial cells critically contribute to CAC formation and progression. ${ }^{11} 16$ Under inflammatory conditions, membrane-bound IL-6R is completely shed off intestinal epithelial cells, becomes soluble and binds IL-6 resulting in enhanced biological IL-6 activity, a process 


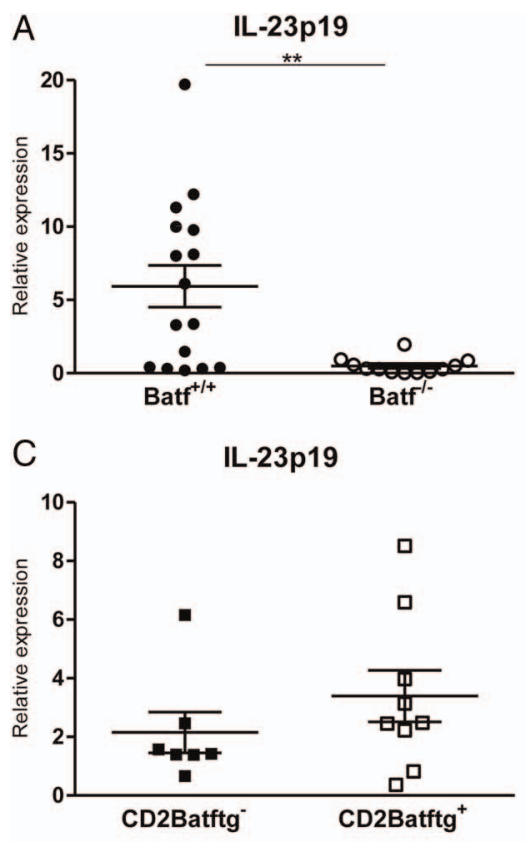

B

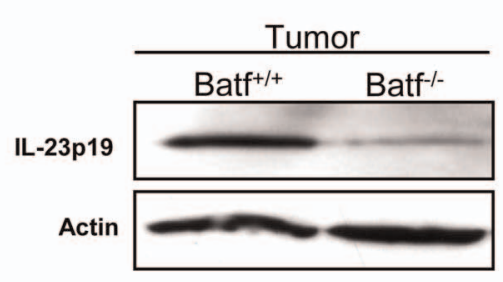

D
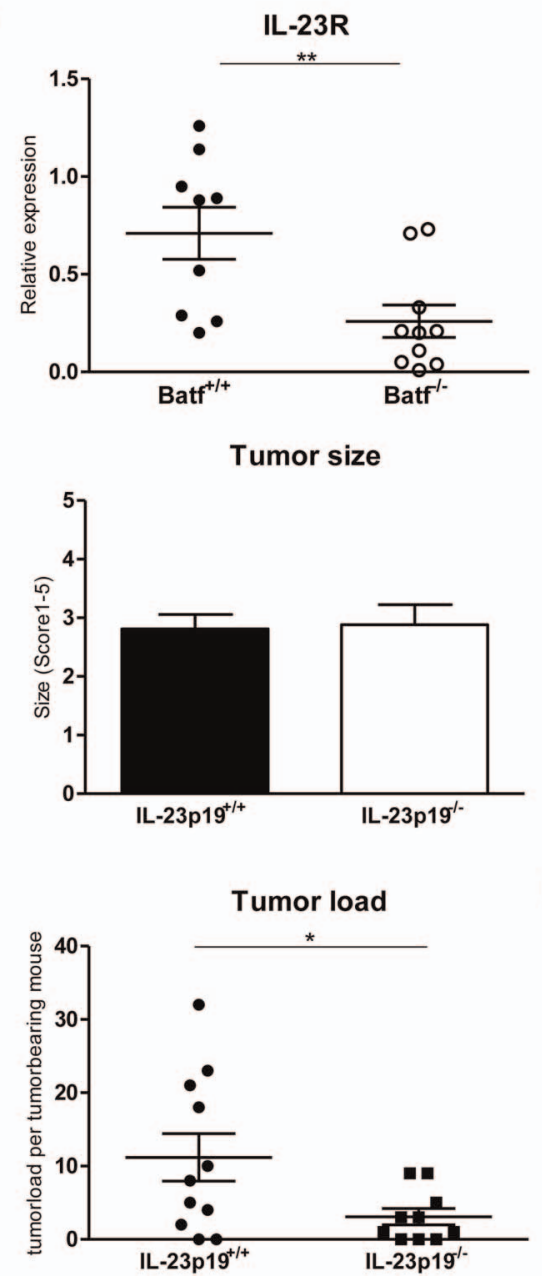

IL-23

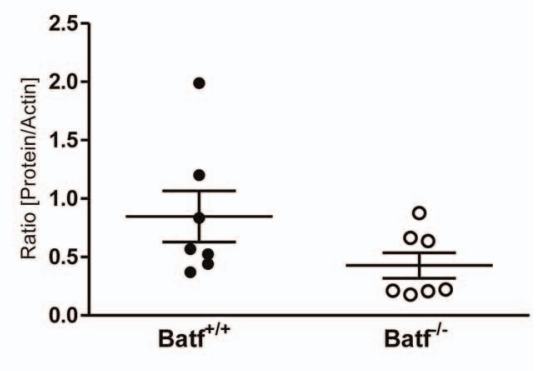

IL-23R

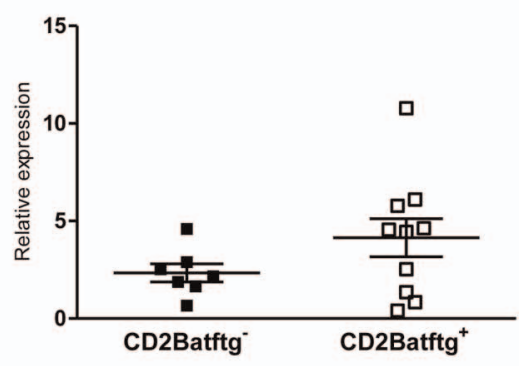

Tumor number

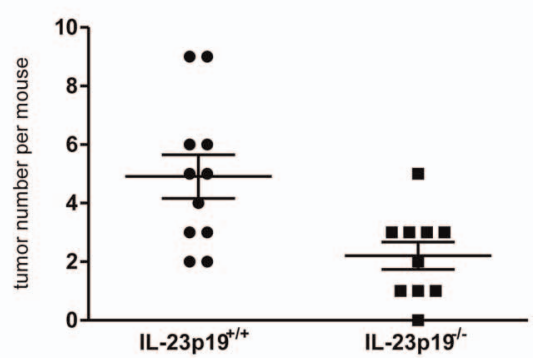

$\mathrm{F}$

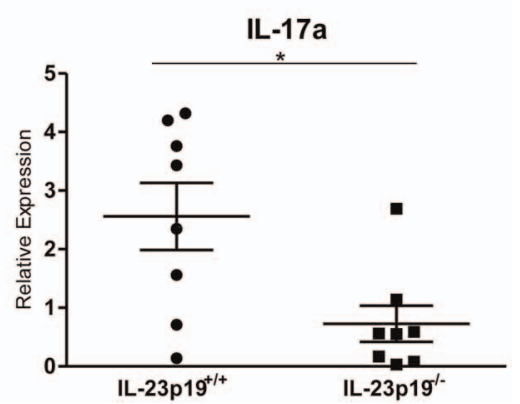

Figure 6 Batf-expressing T cells promote IL-23p19/IL-23R expression in azoxymethan/dextran sodium sulfate (AOM/DSS)-induced colitis-associated cancer tissue. AOM/DSS-treated Batf ${ }^{+/+}$and Batf ${ }^{-1-}$ (A, B, D left panel), CD2Batftg ${ }^{+}$and CD2Batftg ${ }^{-}$(C, D right panel) and IL-23p19 ${ }^{+/+}$and IL-23p1 $19^{-l-}$ mice (E, F), respectively, were observed until day 80. (A), (C) and (D) Quantification of IL-23p19 and IL-23R tumour tissue expression relative to tissue levels of untreated Batf ${ }^{+/+}$or CD2Batftg ${ }^{-}$, respectively, arbitrarily set to 1 by qPCR. (B) IL-23p19 protein detection by western blot within Batf ${ }^{++}$and Batf ${ }^{-1-}$ AOM/DSS-derived tumours (actin=internal control) (left panel); assessment of IL-23R/actin protein expression ratios \pm SEM within normal and colorectal cancer tissues (densitometry) (right panel). (E) Representative endoscopic images displaying polypoid colon tumours $(100 x)$ and assessment of tumour number, size and load of AOM/DSS-treated IL-23p19 $9^{+/+}$and IL-23p19-1- mice ( $\left.\mathrm{n}=10-11 / \mathrm{genotype}\right)$. (F) Quantification of IL-17a tumour tissue expression from IL-23p1 $19^{+/+}$and IL-23p1 $9^{-I-}$ mice as indicated by $\mathrm{PPCR} .{ }^{*} \mathrm{p}<0.05 ;{ }^{* *} \mathrm{p}<0.01 ;{ }^{* * *} \mathrm{p}<0.001$.

specified as IL-6 trans-signalling. ${ }^{22}{ }^{23}$ IL-6 trans-signalling can be mimicked by exogenous administration of Hyper-IL-6, a designer cytokine consisting of linked sIL-6R and IL-6, which was reported to enhance tumour progression in $\mathrm{AOM} /$ DSS-treated, tumour-bearing mice in vivo. ${ }^{11}$ We hypothesised that Hyper-IL- 6 may bypass the necessity of effects normally exerted by IL-23R $\mathrm{R}^{+} \mathrm{IL}-6^{+} \mathrm{CD} 4^{+} \mathrm{T}$ cells and hence reconstitute polypoid tumour progression in $\mathrm{Batf}^{-/-}$mice. To investigate this, $\mathrm{Batf}^{+/+}$and $\mathrm{Batf}^{-/-}$mice were treated with Hyper-IL-6 starting 42 days after initiation of AOM/DSS treatment. Strikingly, Hyper-IL-6 treatment largely reconstituted polyp-shaped tumour development in $\mathrm{Batf}^{-1-}$ mice (figure 8C). Tumour size, tumour number and overall tumour load increased compared with $\mathrm{AOM} / \mathrm{DSS}$-treated $\mathrm{Batf}^{-/-}$control mice (figure 8D). Interestingly, reconstitution of polypoid tumour formation in $\mathrm{Batf}^{-/}$mice in response to Hyper-IL-6 also restored intratumoral IL-23 expression resembling levels within $\mathrm{Batf}^{+/+}$tumours (figure 8E). 
A

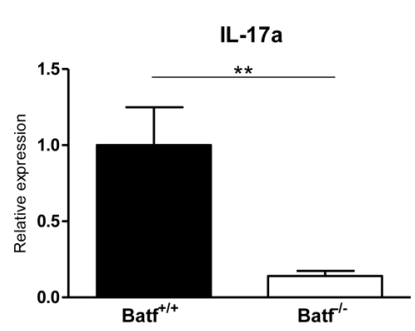

d9

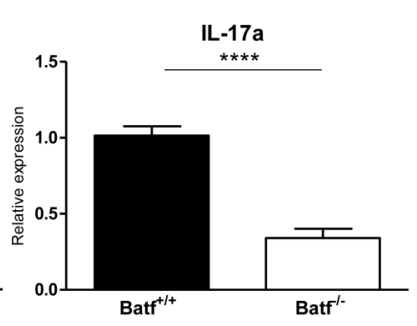

B
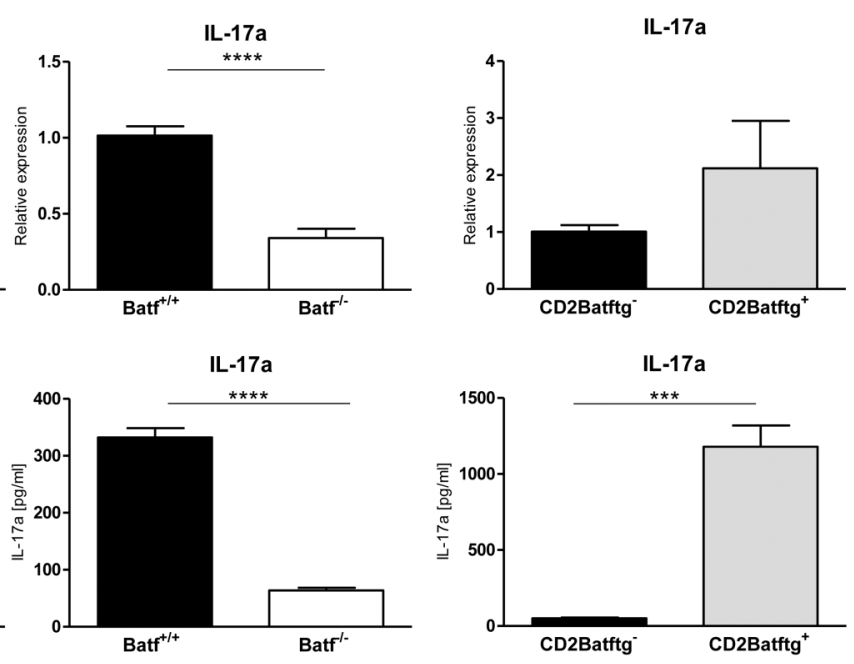

Batf $^{+/+}$

C

d5
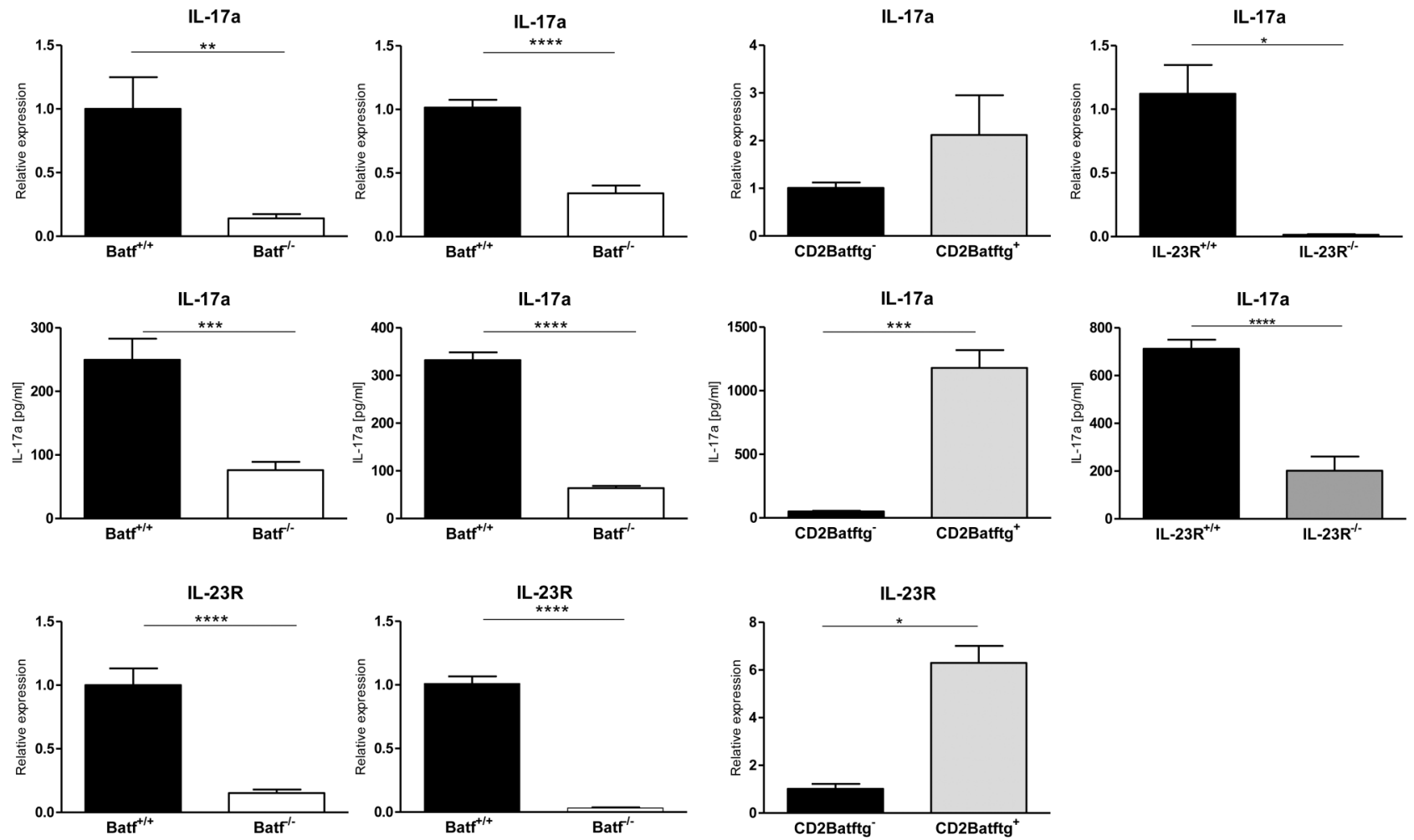

Figure 7 IL-23 induced Th17 development depends on Batf via regulation of the IL-23R expression. Quantitative IL-17a and IL-23R gene expression analyses within IL-1B/IL-6/IL-23-polarised murine CD4 ${ }^{+}$T cells at day 5 (A, left panel) of Batf ${ }^{+/+}$and Batf $^{-/-}$, (B) CD2Batftg ${ }^{-}$and

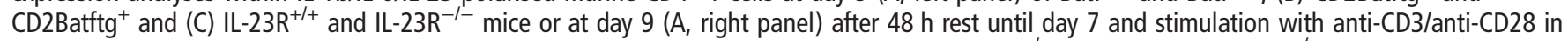
the presence of IL-23 for another $48 \mathrm{~h}$ by qPCR. Data are shown as relative expression with Batf ${ }^{+/+}, \mathrm{CD}_{2} \mathrm{Batftg}^{-}$or IL-23R $^{+/+}$, respectively, expression levels arbitrarily set to 1 . (A-C middle row) Quantitative IL-17a protein analysis (ELISA) in cell-free culture supernatants of indicated CD4 T cells cultured for 5 days or 9 days under IL-23(Th17) conditions as described above. ${ }^{*} p<0.05 ;{ }^{* *} p<0.01 ;{ }^{* * *} p<0.001 ;{ }^{* * * *} p<0.0001$.

\section{DISCUSSION}

Our study identifies Batf-dependent Th17 cells as key regulators of IL-23-driven colon tumour development resolving the longstanding unanswered question as to the cell-type specific contribution of Th17 cells to colon cancer formation. ${ }^{4}$ Batf and interferon regulatory factor 4 were previously shown to orchestrate the regulatory network of Th17 cell differentiation which is further specified by the action of ROR $\gamma$ t. $^{15}$ Our study adds substantial molecular specificity to the protumoral nature of Th17 cells in human disease since we identify Batf but not ROR $\gamma$ t to be a major component of an IL-23/IL-23R ${ }^{+}$molecular network associated with CRC development. Our functional studies in the murine system in two colon tumour models firmly establish that inflammation-induced and sporadic intestinal tumour development is controlled by T cell-intrinsic Batf expression.

Although an IL- $23^{+} / \mathrm{IL}-17 \mathrm{a}^{+}$-driven immune response contributes to CRC progression, investigations targeting the precise contribution of Th17 cells have been hampered by the absence of appropriate model systems. ${ }^{5} 17 \quad 18{ }^{24-26}$ To validate Batf $^{-/-}$ mice as an appropriate in vivo model system to assess the selective contribution of Th17 cells during colon tumour development, we carefully performed a series of experiments and observed that (1) Tumour development was strictly depended on the presence of the $\mathrm{Batf}^{-/-}$lymphocyte compartment. (2) Forced T cell-intrinsic Batf expression resulted in enhanced Th17 development and increased tumour formation. (3) Development and functionality of IL-17a-/IL-22-producing non-Th17 immune cell subsets were normal in $\mathrm{Batf}^{-1-}$ mice in striking contrast to a series of studies employing mice with germline deletions of Th17 related cytokines or other transcriptional regulators like ROR $\gamma \mathrm{t} .{ }^{24-26}$ Collectively, this data provide sufficient evidence to support our conclusion that reduced colon tumour formation in $\mathrm{Batf}^{-/-}$mice results from the absence of Th17 cells.

Our study also yielded the unexpected, to our best knowledge unparalleled result of a dissociation between IL-23 and IL-17a expression in $\mathrm{Batf}^{-1-}$ tumours. In the AOM/DSS model IL-23p $19^{-/-}$mice showed reduced CAC colon tumour formation along with reduced IL-17a tissue expression in agreement with results previously described in a sporadic colon tumour model. ${ }^{18} 27$ While these results imply that IL-23 and IL-17a are coregulated, our findings employing the $\mathrm{Batf}^{-/-}$mouse model however object the interpretation that IL-17a alone is sufficient to promote colon tumour formation and point towards alternative IL-23-dependent protumoral pathways. Cellular targets of IL-23 in the context of colon cancer have not been described but IL-23-induced effects are presumably mediated by an indirect mechanism involving IL-23R-expressing immune subsets. ${ }^{27}$ In contrast to sporadic colon tumour tissue, IL-23R expression levels within murine CAC samples were significantly upregulated suggesting differences in the composition and functionality of the immune cell infiltrate present in these colon tumour types. Interestingly, IL-23R expression levels in $\mathrm{Batf}^{-/-}$colon tumour tissue were significantly reduced. Given our results of Batf-dependent IL-23R expression by Th17 cells in vitro, reduced IL-23R expression by intratumoral $\mathrm{Batf}^{-/-} \mathrm{CD}^{+}$ 
A

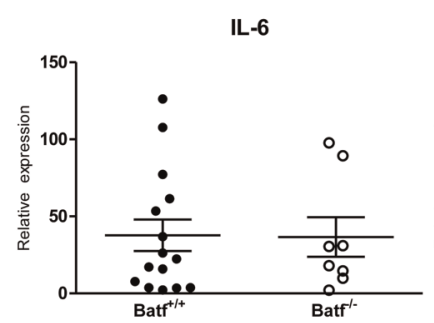

B

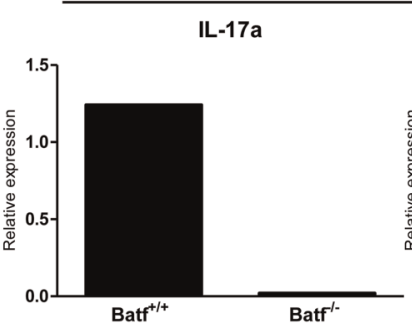

$\mathrm{CD}^{+}{ }^{+} \mathrm{TIL}$

IL-23R

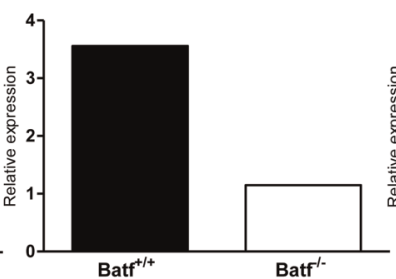

IL-6

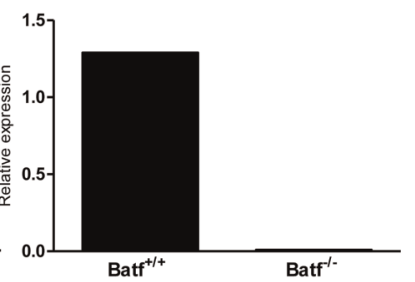

C Colonoscopy $\mathrm{NBI}$
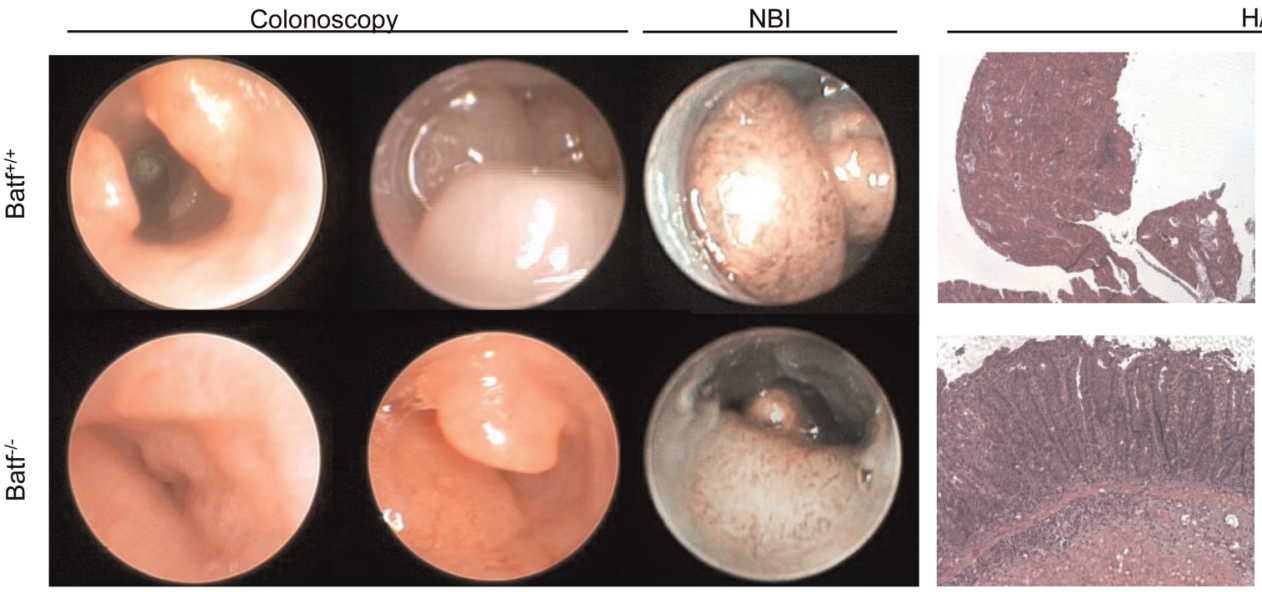
H/E E

\section{Hyper-IL6}

D

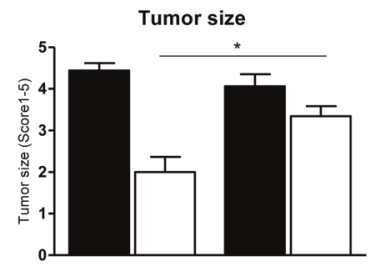

Hyper-IL6
$+$

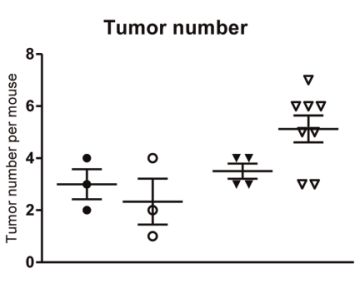

$+$

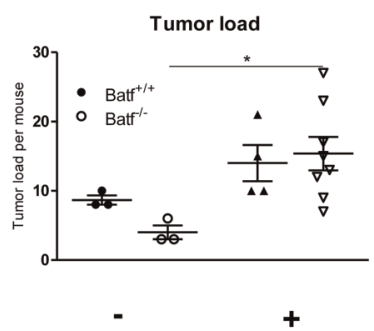

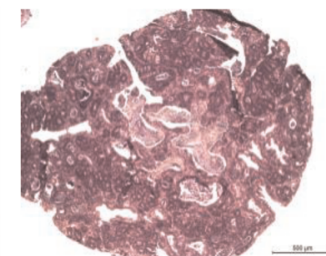

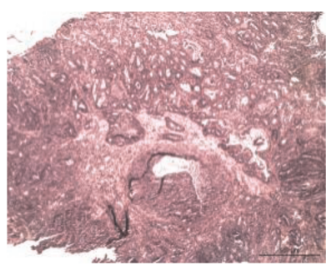

$+$

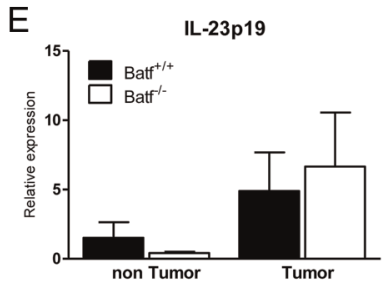

Figure $8 \mathrm{IL}-6 / \mathrm{sIL}-6 \mathrm{R}$ treatment restores polypoid colitis-associated cancer (CAC) progression in Batf ${ }^{-/}$mice. Batf ${ }^{+/+}$and Batf ${ }^{-/}$mice were treated with azoxymethan/dextran sodium sulfate (AOM/DSS). (A) Quantification of IL-6 gene expression within polypoid (Batf ${ }^{+/+}: n=16 ; B^{2}$ Baff $^{-1-}: n=9$ ) colon tumours by qPCR (one-way ANOVA test; ${ }^{* *} \mathrm{p}<0.01$ ). (B) Tumour-infiltrating CD4 $4^{+}$lamina propia (LP) T cells of AOM/DSS-treated Batf ${ }^{+/+}$and Batf ${ }^{-1-}$ mice ( $n=5-10$ mice each, pooled) were sort-purified in $\mathrm{CD}^{+}$and $\mathrm{CD} 4^{-}$LP cells. Quantification of IL-17a, IL-23R and IL-6 expression by $\mathrm{CD} 4^{+}$cells via QPCR. Data show one representative experiment. (C) 42 days after initiation of AOM/DSS-treatment Batf ${ }^{+/+}$and Batf ${ }^{-/}$mice were either left

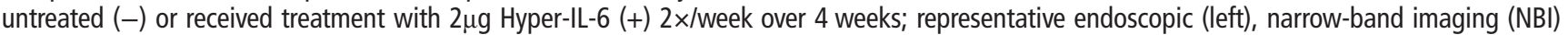
(middle) and H\&E (right) (scale bar=500 $\mu \mathrm{m}$ ) images. (D) Size, number and load \pm SEM of polypoid tumours (-) or (+) Hyper-IL-6 treatment (Batf ${ }^{+/}$ ${ }^{+} \mathrm{h}-\mathrm{IL}-6 \mathrm{n}=4$; Batf ${ }^{-I} \mathrm{~h}-\mathrm{IL}-6 \mathrm{n}=8$ ). (E) Quantification of IL-23p19 gene expression in normal colon and CAC tissues after Hyper-IL-6 (+) treatment at day 70. Data represent pooled results from two independent experiments shown as mean values \pm SEM. ${ }^{*} p<0.05$.

$\mathrm{T}$ cells and normal IL-23 responsiveness of $\mathrm{Batf}^{-/-} \mathrm{TCR} \gamma / \delta^{+}$ $\mathrm{T}$ cells and ILC3 cells, we conclude that reduced intratumoral IL-23R expression in $\mathrm{Batf}^{-/-}$mice reflects abrogated IL-23R expression by $\mathrm{CD}^{+}{ }^{+} \mathrm{T}$ cells. Collectively, our data place Batf-dependent IL-23R ${ }^{+}$Th17 cells in the centre of the pathogenesis of murine colon tumour development.

The IL-6/Stat-3 pathway was previously shown to critically contribute to CAC development and progression. ${ }^{11}{ }^{16} \mathrm{IL}-6$ is produced by intratumoral T cells and CD11c ${ }^{+}$APCs. ${ }^{11} 2228$ Our finding that IL-6 expression by $\mathrm{CD}^{+}$but not $\mathrm{CD} 4^{-} \mathrm{TIL}$ is Batf-dependent is striking since $T$ cells and IL-6/Stat-3 peak expression are predominately located at the interface of immune and tumour cells implying a close interaction. ${ }^{11} 2829$ Further, in the B16 melanoma model Stat-3 signalling was shown to directly induce IL-23 expression in tumour-associated APC leading to a shift within the tumour microenvironment towards unfavourable IL-23 expression. ${ }^{30}$ Our results in the AOM/DSS model suggest that $\mathrm{T}$ cell-derived IL- 6 might be the crucial switch locally regulating IL-23 expression resulting in CAC progression. This hypothesis is strongly supported by our experimental finding that Hyper-IL-6 administration restored polypoid tumour development in AOM/DSS-treated Batf $^{-/}$mice. ${ }^{11}$ Importantly, Hyper-IL-6 also restored IL-23 tissue expression in Batf $^{-/}$tumours experimentally excluding a primary IL-23 expression deficiency of $\mathrm{Batf}^{-/-}$APCs.

Together, our study identified Batf-dependent Th17 cells as crucial regulators of sporadic as well as inflammation-induced colon tumour development. Mechanistically, we discovered tumour-infiltrating Batf-dependent IL-23R ${ }^{+} \mathrm{CD} 4^{+} \mathrm{T}$ cells to be critical for the establishment of an IL-23-enriched tumour microenvironment. Our data suggest that T cell-derived IL-6 represents the critical molecular link between IL-23R expression by $T C R \alpha \beta^{+} T$ cells and tumour-associated IL-23 expression required for CAC progression. In our proposed model the 
T cell/APC interaction installs a positive feedback loop progressively expanding IL-23R ${ }^{+} \mathrm{T}$ cells in response to IL-23 and potentially IL-6 signalling while IL-23 expression by APC is induced and maintained by $\mathrm{T}$ cell-derived IL- 6 -induced Stat-3 signalling (see online supplementary figure S16). ${ }^{1122}$ Strikingly, we identified Batf $^{+}$IL-23R ${ }^{+}$IL- $6{ }^{+}$CD $4^{+} \mathrm{T}$ cells within human CRC tissue that might represent the human equivalent of the Batf-dependent $\mathrm{T}$ cell subpopulation associated with tumour progression in mice. Hence, Batf-dependent IL-23R ${ }^{+} \mathrm{T}$ cells represent a potential future therapeutic target limiting CRC progression.

\section{Author affiliations} \\ ${ }^{1}$ Department of Medicine 1, University Hospital Erlangen, University of Erlangen- \\ Nuremberg, Kussmaul Campus for Medical Research, Erlangen, Germany \\ ${ }^{2}$ Max Eder Research Group supported by the German Cancer Aid, University Hospital \\ Erlangen, Erlangen, Germany \\ ${ }^{3}$ Department of Nephropathology, Institute of Pathology, University Hospital \\ Erlangen, Erlangen, Germany \\ ${ }^{4}$ Department of Surgery, University Hospital, Erlangen, Germany \\ ${ }^{5}$ Institute of Biochemistry, Christian-Albrechts-University, Kiel, Germany \\ ${ }^{6}$ Medical Department, Hospital Ostholstein, Eutin, Germany \\ ${ }^{7}$ Department of Gastroenterology, Endocrinology and Metabolism, University Hospital \\ of Marburg, Philipps-University of Marburg, Marburg, Germany
}

Acknowledgements The authors thank Jana Petzold and Irena Klaußner for excellent technical assistance. The authors thank the 'Cell Sorting and Immunomonitoring' Core Unit at the Department of Dermatolgy, University Hospital Erlangen, for excellent assistance.

Contributors EP did experiments together with TV; MW, RA, BA, JM, SM, EG, SR-J and MFN provided clinical samples, protocols, reagents or designed experiments; EP, TV, MB, KA and KH analysed data; EP, TV, MW, BA, MB, KA, SR-J, MFN and KH discussed and interpreted findings; $\mathrm{KH}$ directed the work and wrote the manuscript.

Funding This study was supported by The Max-Eder Research Program of the German Cancer Aid project \#109307 (KH), the ELAN programmes (project \#09.08.19.1) of the University Erlangen-Nuremberg (KH), the Interdisciplinary Center for Clinical Research Erlangen, project A53, $(\mathrm{KH})$ and the Clinical Research Group CEDER of the German Research Council (RA and MFN). SRJ was supported by grants from the Deutsche Forschungsgemeinschaft Bonn, Germany (SFB841, project C1; SFB877 project A1) and by the Cluster of Excellence 'Inflammation at Interface'. EG was supported by a grant from the Deutsche Forschungsgemeinschaft (Ga762/3-2).

Competing interests None.

Ethics approval Inhouse ethical commission University Hospital Erlangen.

Provenance and peer review Not commissioned; externally peer reviewed.

\section{REFERENCES}

1 Danese $S$, Mantovani A. Inflammatory bowel disease and intestinal cancer: a paradigm of the Yin-Yang interplay between inflammation and cancer. Oncogene 2010;29:3313-23.

2 Strober W, Fuss I, Mannon P. The fundamental basis of inflammatory bowel disease. J Clin Invest 2007;117:514-21.

3 Fridman WH, Pages F, Sautes-Fridman $\mathrm{C}$, et al. The immune contexture in human tumours: impact on clinical outcome. Nat Rev Cancer 2012;12:298-306.

4 De Simone V, Pallone F, Monteleone G, et al. Role of T17 cytokines in the control of colorectal cancer. Oncoimmunology 2013;2:e26617.
5 Tosolini M, Kirilovsky A, Mlecnik B, et al. Clinical impact of different classes of infiltrating T cytotoxic and helper cells (Th1, th2, treg, th17) in patients with colorectal cancer. Cancer Res 2011;71:1263-71.

6 Schraml BU, Hildner K, Ise W, et al. The AP-1 transcription factor Batf controls $T(H)$ 17 differentiation. Nature 2009;460:405-9.

7 El-Behi M, Ciric B, Dai H, et al. The encephalitogenicity of $T(H) 17$ cells is dependent on IL-1- and IL-23-induced production of the cytokine GM-CSF. Nat Immunol 2011;12:568-75.

8 Spits H, Artis D, Colonna M, et al. Innate lymphoid cells - a proposal for uniform nomenclature. Nat Rev Immunol 2013;13:145-9.

9 Szabo SJ, Sullivan BM, Stemmann C, et al. Distinct effects of T-bet in TH1 lineage commitment and IFN-gamma production in CD4 and CD8T cells. Science 2002;295:338-42.

10 Becker C, Dornhoff H, Neufert C, et al. Cutting edge: IL-23 cross-regulates IL-12 production in T cell-dependent experimental colitis. J Immuno/ 2006;177:2760-4.

11 Grivennikov S, Karin E, Terzic J, et al. IL-6 and Stat3 are required for survival of intestinal epithelial cells and development of colitis-associated cancer. Cancer Cell 2009;15:103-13.

12 Skarnes WC, Rosen $B$, West $A P$, et al. A conditional knockout resource for the genome-wide study of mouse gene function. Nature 2011;474:337-42.

13 Fujino S, Andoh A, Bamba S, et al. Increased expression of interleukin 17 in inflammatory bowel disease. Gut 2003;52:65-70.

14 Kobayashi T, Okamoto S, Hisamatsu T, et al. IL23 differentially regulates the Th1/Th17 balance in ulcerative colitis and Crohn's disease. Gut 2008;57:1682-9.

15 Ciofani M, Madar A, Galan C, et al. A validated regulatory network for Th17 cell specification. Cell 2012;151:289-303.

16 Bollrath J, Phesse TJ, von Burstin VA, et al. gp130-mediated Stat3 activation in enterocytes regulates cell survival and cell-cycle progression during colitis-associated tumorigenesis. Cancer Cell 2009;15:91-102.

17 Langowski JL, Zhang $X$, Wu L, et al. IL-23 promotes tumour incidence and growth. Nature 2006;442:461-5.

18 Grivennikov SI, Wang K, Mucida D, et al. Adenoma-linked barrier defects and microbial products drive IL-23/IL-17-mediated tumour growth. Nature 2012;491:254-8.

19 Neufert C, Becker C, Neurath MF. An inducible mouse model of colon carcinogenesis for the analysis of sporadic and inflammation-driven tumor progression. Nat Protoc 2007;2:1998-2004.

20 Sutton CE, Lalor SJ, Sweeney CM, et al. Interleukin-1 and IL-23 induce innate IL-17 production from gammadelta T cells, amplifying Th17 responses and autoimmunity. Immunity 2009;31:331-41.

21 Stockinger B, Veldhoen M. Differentiation and function of Th17T cells. Curr Opin Immunol 2007;19:281-6.

22 Becker C, Fantini MC, Schramm C, et al. TGF-beta suppresses tumor progression in colon cancer by inhibition of IL-6 trans-signaling. Immunity 2004;21:491-501.

23 Lertkhachonsuk AA, Yip CH, Khuhaprema T, et al. Cancer prevention in Asia: resource-stratified guidelines from the Asian Oncology Summit 2013. Lancet Oncol 2013;14:e497-507.

24 Hyun YS, Han DS, Lee AR, et al. Role of IL-17A in the development of colitis-associated cancer. Carcinogenesis 2012;33:931-6.

25 Tong Z, Yang XO, Yan $\mathrm{H}$, et al. A protective role by interleukin-17F in colon tumorigenesis. PLOS ONE 2012;7:e34959.

26 Eberl G, Marmon S, Sunshine MJ, et al. An essential function for the nuclear receptor RORgamma(t) in the generation of fetal lymphoid tissue inducer cells. Nat Immunol 2004;5:64-73.

27 Wang K, Grivennikov SI, Karin M. Implications of anti-cytokine therapy in colorectal cancer and autoimmune diseases. Ann Rheum Dis 2013;72(Suppl 2):ii100-3.

28 De Simone V, Franze E, Ronchetti G, et al. Th17-type cytokines, IL-6 and TNF-alpha synergistically activate STAT3 and NF-kB to promote colorectal cancer cell growth. Oncogene 2014. http://dx.doi.org/10.1038/onc.2014.286

29 Bromberg J, Wang TC. Inflammation and cancer: IL-6 and STAT3 complete the link. Cancer Cell 2009;15:79-80.

30 Kortylewski M, Xin H, Kujawski M, et al. Regulation of the IL-23 and IL-12 balance by Stat3 signaling in the tumor microenvironment. Cancer Cell 2009;15:114-23. 


\section{GUT Batf-dependent Th17 cells critically regulate IL-23 driven colitis-associated colon cancer}

Elise Punkenburg, Tina Vogler, Maike Büttner, Kerstin Amann, Max Waldner, Raja Atreya, Benjamin Abendroth, Jonas Mudter, Susanne Merkel, Eike Gallmeier, Stefan Rose-John, Markus F Neurath and Kai Hildner

Gut2016 65: 1139-1150 originally published online April 2, 2015 doi: 10.1136/gutjnl-2014-308227

Updated information and services can be found at:

http://gut.bmj.com/content/65/7/1139

These include:

Supplementary Supplementary material can be found at:

Material http://gut.bmj.com/content/suppl/2015/04/04/gutjnl-2014-308227.DC1

References This article cites 29 articles, 6 of which you can access for free at: http://gut.bmj.com/content/65/7/1139\#ref-list-1

\section{Email alerting}

Receive free email alerts when new articles cite this article. Sign up in the service box at the top right corner of the online article.

Collections

Articles on similar topics can be found in the following collections

\section{Notes}

To request permissions go to:

http://group.bmj.com/group/rights-licensing/permissions

To order reprints go to:

http://journals.bmj.com/cgi/reprintform

To subscribe to BMJ go to:

http://group.bmj.com/subscribe/ 